\title{
Observables for possible QGP signatures in central $p p$ collisions
}

\author{
Benjamin Nachman $^{1, a}$, Michelangelo L. Mangano ${ }^{2}$ \\ ${ }^{1}$ Physics Division, Lawrence Berkeley National Laboratory, Berkeley, CA 94704, USA \\ 2 Theoretical Physics Department, CERN, 1211 Geneva 23, Switzerland
}

Received: 3 October 2017 / Accepted: 21 April 2018 / Published online: 28 April 2018

(C) The Author(s) 2018

\begin{abstract}
Proton-proton ( $p p$ ) data show collective effects, such as long-range azimuthal correlations and strangeness enhancement, which are similar to phenomenology observed in heavy ion collisions. Using simulations with and without explicit existing models of collective effects, we explore new ways to probe $p p$ collisions at high multiplicity, in order to suggest measurements that could help identify the similarities and differences between large- and small-scale collective effects. In particular, we focus on the properties of jets produced in ultra-central $p p$ collisions in association with a $Z$ boson. We consider observables such as jet energy loss and jet shapes, which could point to the possible existence of an underlying quark-gluon plasma, or other new dynamical effects related to the presence of large hadronic densities.
\end{abstract}

\section{Introduction}

There has been a recent surge of interest in collective effects in small systems with high final state multiplicity due to measurements of strangeness enhancement from ALICE [1] and large-angle particle correlations (the 'ridge') by ATLAS [2] and CMS [3,4]. These effects are not reproduced by the standard Monte Carlo (MC) event generators for $p p$ collisions, based on standard Quantum Chromodynamics (QCD) evolution and well-tested models of hadronization [5-11]. The features of these phenomena resemble those exhibited by the Quark Gluon Plasma (QGP) formed in heavy ion (HI) collisions. If parameterized in terms of $d N / d \eta$, the evolution of the observed effects with $d \mathrm{~N} / d \eta$ in $p p$ smoothly matches to the size of the effects observed in HI collisions, where they are interpreted in terms of QGP dynamics (see e.g. Fig. 2 in Ref. [1]). It is therefore tempting to speculate that a sort of "miniQGP" might be formed in (or might be responsible for) the highest $d N / d \eta$ events in $p p$. Alternative interpretations have nevertheless been put forward, relying on a more complex

a e-mail: bnachman@cern.ch description of the fragmentation phase of the event generation [12-14]. These descriptions of the collective phenomena make no reference to a QGP, and derive their results from a more extended network of interactions among the partons emerging from the usual $(T=0)$ evolution of the partonic final state. More generally, the experimental facts raise the question of whether the description of large-multiplicity final states in $p p$ collisions boils down to finding the right knobs to tune in some fragmentation model, or whether it requires the understanding of a new dynamical phase of high-energy hadronic interactions.

In this paper we propose a set of observables that, while being sensitive to the reported collective effects, would likely lead to different results depending on whether the QGP is active or not. In particular, we consider jet observables, which in the presence of a QGP are expected to undergo quenching effects that may not exist in non-QGP models of collective effects in $p p$. We analyze $Z+$ jet events, and study the properties of the jets and of the surrounding environment, as a function of the track multiplicity. We focus on both the strangeness enhancement and on the potential quenching of the jet recoiling against the $Z$ boson. We show that the MC models predicting strange enhancement in high-multiplicity minimum bias events continue exhibiting large differences in the modeling of strange hadron production, with respect to the standard MCs. We also show, perhaps not surprisingly, that those models do not lead to an observable quenching of the jet energy, and an observable such as $p_{\mathrm{T}, J} / p_{\mathrm{T}, Z}$ shows no significant dependence on $d N / d \eta$, matching the prediction of MCs that do not model collective effects.

We suggest that the experimental study of strangeness enhancement and quenching in $Z$ (or $\gamma$ ) plus jet events might help in better assessing the true nature of the collective phenomena recently observed in $p p$ collisions, proving or disproving their QGP-like origin, and providing valuable data to improve the MC models and their tuning. Studies in $p P b$ by ATLAS [15-26], CMS [27-41], ALICE [42-80], and LHCb [81-86] have also started to probe an intermediate regime 
between $p p$ and $P b P b$, though with limited datasets compared with $p p$.

This paper is organized as follows. Section 2 describes the simulation setup before the results are presented in Sect. 3. Inclusive strangeness enhancement is demonstrated in Sect. 3.1 in addition to probing strangeness inside jets. Momentum balancing is investigated in Sect. 3.2 and additional observables related to jet substructure are studied in Sect. 3.3. We present our conclusions in Sect. 4.

\section{Simulation}

\subsection{Collective effects}

There have been several attempts within the context of the string hadronization model [87] to describe collective effects. In string hadronization, color flux tubes ('strings') connecting partons produce a linearly confining potential. Kinks in the string describe gluons and strings can break into $q q^{\prime}$ pairs; various phenomenological and physics-inspired parameters determine the distribution of hadrons produced.

One very promising extension that provides an excellent description of strangeness enhancement observed by ALICE [1] is the rope hadronization model. Rope hadronization extends the string picture by allowing nearby strings to interact coherently to have an effectively higher string tension. Due to their larger masses, strange hadrons are suppressed with respect to purely $u / d$ hadrons by $\sim m_{s}^{2} / \kappa$, where $\kappa$ is the string tension $\kappa \sim \mathcal{O}(1) \mathrm{GeV} / \mathrm{fm}$. Ropes can have a higher tension than single strings, which is what gives rise to strangeness enhancement. This higher tension can also affect other aspects of fragmentation, though as will be true for all the models described in this section, observables that are largely insensitive to hadronization may not be very sensitive to this modification. ${ }^{1}$ We use the rope hadronization plugin [88] to Pythia $8.226[5,10]$ to model collective effects with ropes. ${ }^{2}$

While the rope hadronization model is an explicit attempt to describe some collective effects, color reconnection (CR) models that attempt to model $1 / N_{\mathrm{c}}^{2}$ effects beyond the naive $N_{\mathrm{c}}=\infty$ limit can also result in collective phenomena. For example, the default CR model in Pythia 6 [5] has been shown to produce flow-like effects in $p p$ collisions [91]. This has also been demonstrated [92] for a more recent QCD-inspired

\footnotetext{
${ }_{1}^{1}$ In particular, this means that if these models do not predict jet modification, we cannot rule out such effects at the LHC - none of these models currently explains all observed collective effects in $p p$. However, they serve as useful benchmarks.

2 This model does not include shoving [89], which has been shown to qualitatively explain the ridge. Recent work to incorporate shoving into Pythia 8 is promising and would provide an additionally useful benchmark for jet studies in the future when it is ready [90].
}

model of color reconnection [93]. Both the new and original $\mathrm{CR}$ models are studied by either switching to the new model or turning off CR completely.

A third approach to collective effects draws inspiration from thermodynamics [14]. Nearby strings increase an effective temperature that describes the distribution of hadrons and their momentum spectra. This model also includes rescattering (secondary parton collisions after the first one), which is inspired by the formation of a dense hadronic gas when the string density is sufficiently high.

The above models are simulated by modifying Pythia $8.226[5,10]$ with the default tune [94] for $p p$ collisions at ${ }^{3}$ $\sqrt{s}=7 \mathrm{TeV}$. None of the models were explicitly developed or tested for $p p \rightarrow Z+$ jets, but they can still provide useful benchmarks. Other simulators have been designed to incorporate collective effects, such as Dipsy [95] and EPOS LHC [13], but they can only simulate minimum bias events. The rope hadronization model will be used as the prototypical model of collective effects and the other Pythia modifications will be shown as well. Appendix A documents all of the parameters used for the various models.

\subsection{Event reconstruction}

The $Z$-boson is required to decay into muons and $\mid m_{\mu \mu}-$ $m_{Z} \mid<15 \mathrm{GeV}$. Stable particles $(c \tau \leq 10 \mathrm{~mm})$ excluding muons and neutrinos are clustered into jets with FastJet 3.1.3 [96] using the anti- $k_{t}$ algorithm [97] with a jet radius of $R=0.4$. Unstable strange hadrons are assigned to jets via ghost association [98]. Jet catchment areas are calculated using the median area from the Voronoi method applied to $k_{t}$ jets clustered from particles out to $|\eta|=2$. Signal jets are required to have $p_{\mathrm{T}}>20 \mathrm{GeV}$ and $|\eta|<2$. For some technical plots below we shall also use 'soft jets', with 10 $\mathrm{GeV}<p_{\mathrm{T}}<20 \mathrm{GeV}$ (too low to be reconstructed in practice). All events are required to have exactly one signal jet and $\mid \Delta \phi($ jet, $Z) \mid>1$ rad to reduce the likely presence of a second jet that is below threshold. In $\mathrm{HI}$ and $\mathrm{pPb}$ collisions, the 'centrality' of an event is often quantified by the number of particles measured in the event. Therefore, we study event and jet properties as a function of the measured multiplicity. There are many ways to quantify the multiplicity:

1. Total track multiplicity (TTM). General purpose detectors like ATLAS and CMS have tracking coverage up to $|\eta|<2.5$ and $p_{\mathrm{T}} \gtrsim 200 \mathrm{MeV}$. Tracks are excluded

\footnotetext{
${ }^{3}$ We focus on $7 \mathrm{TeV}$ since this is the LHC dataset with the lowest pileup, and is more suitable for the type of analysis discussed here. However, if one restricts to track-only observables, the high pileup data may also be usable by only looking at the tracks associated to a single vertex.
} 


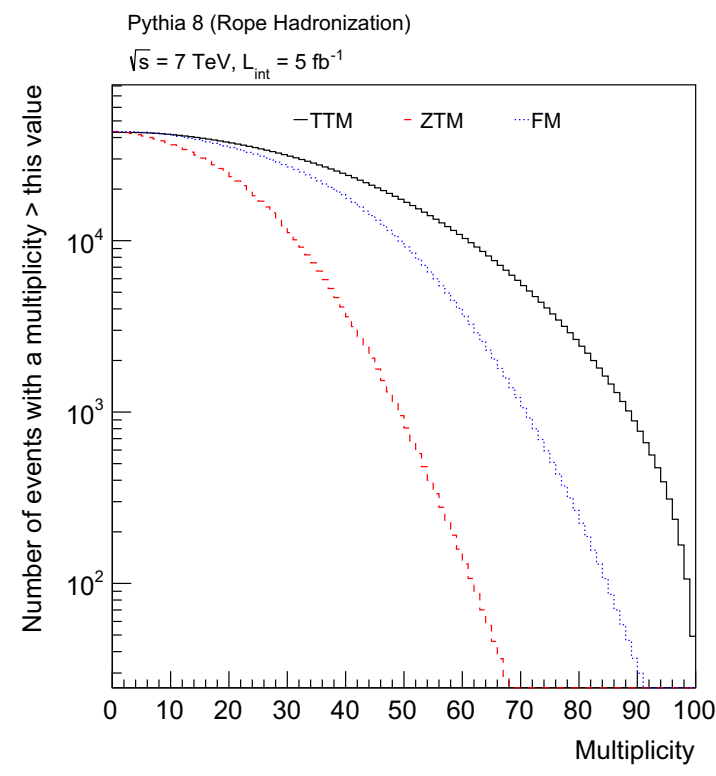

Fig. 1 Left: The number of expected $Z+1$ jet events passing the selection cuts, in $5 \mathrm{fb}^{-1}$, with a given multiplicity threshold defined by TTM, ZTM, or FM. Right: the number of events that pass a threshold on

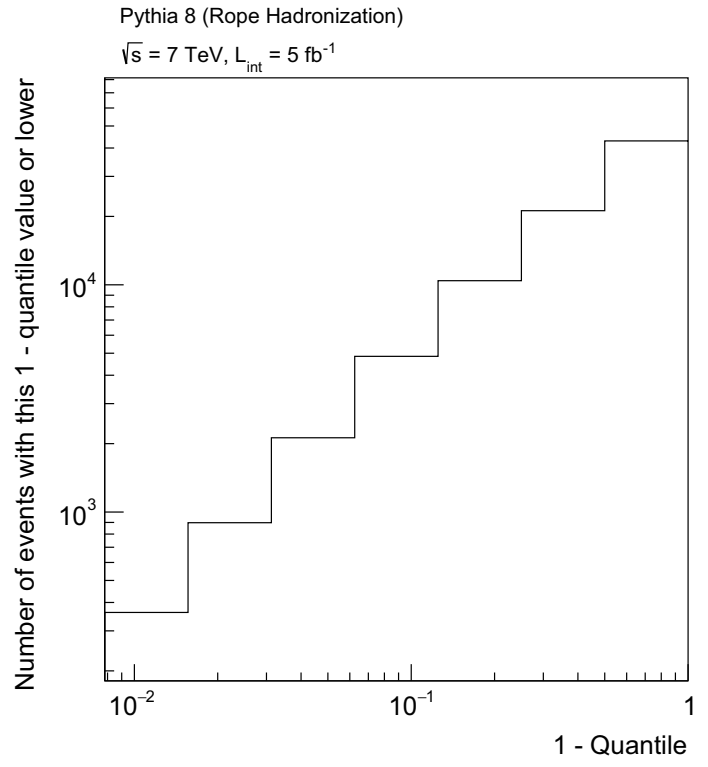

the multiplicity quantile (the same by construction for all multiplicity types). Higher multiplicities are on the left

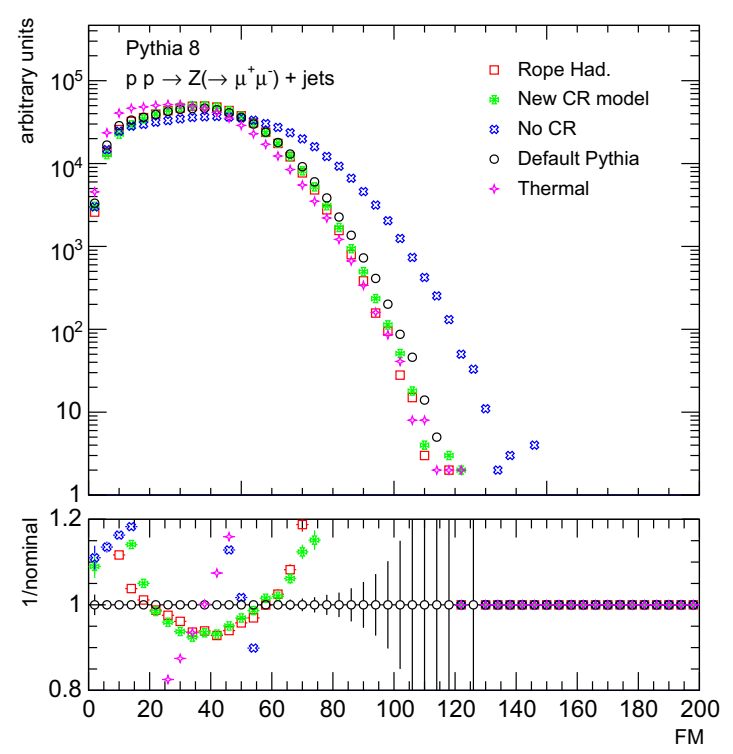

Fig. 2 Left: The distribution of the three definitions of multiplicity before for the default and Rope hadronization models. Right: a comparison of the FM for the various models described in Sect. 2.1

if they are within an annulus of $\Delta R<0.6$ around the signal jet axis.

2. Z-side track multiplicity (ZTM). Despite an annulus cut around the jet axis, the TTM may be biased by the presence of a jet due to large angle radiation from the parton(s) recoiling from the $Z$. One way around this is to count the number of tracks that are in the $Z$ boson hemisphere defined by $\cos (\Delta \phi(Z$, track $))>0$.
3. Forward Multiplicity (FM). Even if the tracks from the jet side are removed, the hard $Q^{2}$ process can still influence the central multiplicity. Therefore, the number of very forward particles can be used as measure of event activity. This is a tradeoff between sensitivity to the underlying event activity that might influence the hard $Q^{2}$ process and a potential bias from the hard $Q^{2}$ process itself influencing the multiplicity. We use a cutoff of $4<|\eta|<5$, 


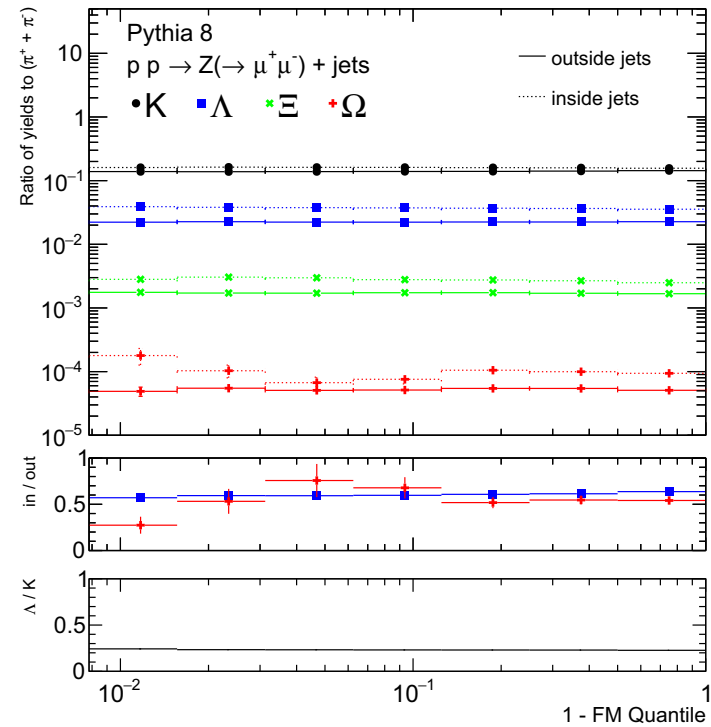

Fig. 3 The relative abundance of strange hadrons as a function of the FM quantile. Low values of the $x$-axis correspond to extreme multiplicities; the rightmost bin captures events with multiplicities at or

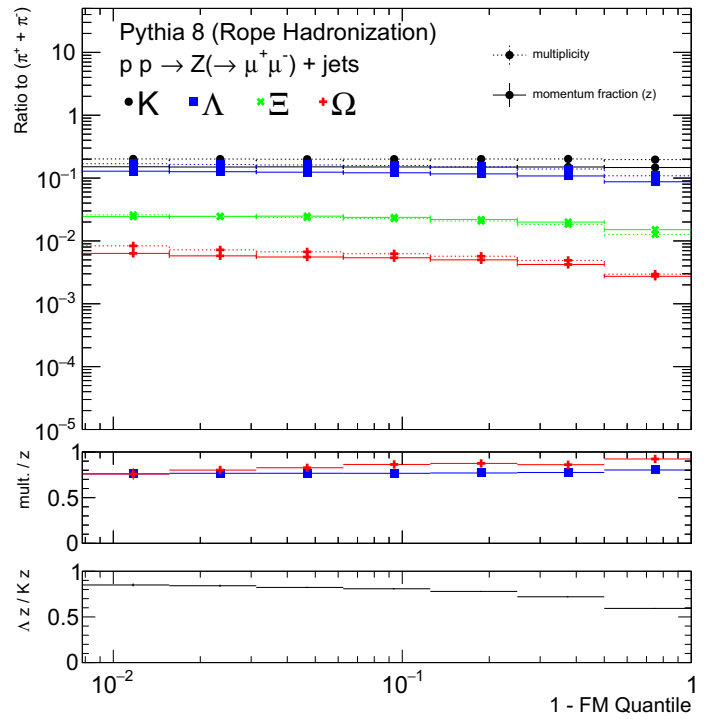

Fig. 4 Same as Fig. 3, but comparing the multiplicity inside jets with the average momentum fraction carried by those hadrons

which is consistent with the ALICE forward scintillators [99] and ATLAS/CMS forward calorimeters [100,101].

Figure 1 shows the number of predicted events with a multiplicity defined by TTM, ZTM, and FM for the Rope hadronization model. One key advantage of $Z$ (or $\gamma)+$ jets in $p p$ collisions versus $p P b$ is that the integrated luminosities collected by ATLAS and CMS of the former are much larger than all four experiments' datasets for the latter. With only the $\sqrt{s}=7 \mathrm{TeV}$ dataset, of about $5 \mathrm{fb}^{-1}$, there are many

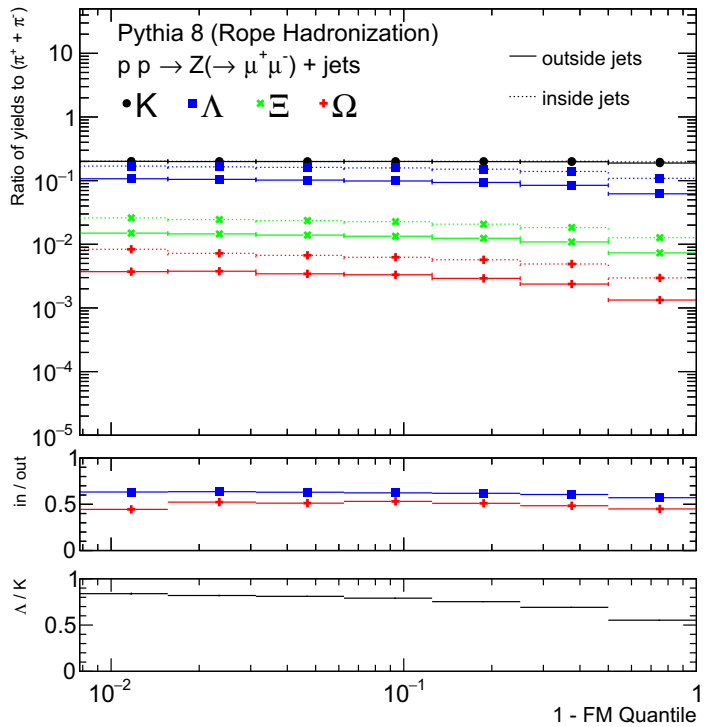

below the median FM. The left plot shows the trends without the Rope hadronization model while the right plot has the Rope model enabled

hundreds of $Z \rightarrow \mu^{+} \mu^{-}$events with a single jet that are in the $>99 \%$ percentile of the multiplicity distribution. The $n$th quantile is defined such that there are a fraction $n$ of events that have this multiplicity or smaller. It is a useful notion for normalizing the multiplicity to make direct comparisons between definitions. Events in the $99 \%$ percentile are such that only $1 \%$ of events have a higher multiplicity.

The actual multiplicity distributions for the three definitions are shown in Fig. 2. By construction, ZTM is less than or equal to the TTM and is typically a factor of two smaller. The Rope hadronization model predicts a different multiplicity distribution than the nominal; to control for any effects to these differences when comparing observables in multiplicity ranges, the multiplicity distribution for the standard hadronization is re-weighted to match the Rope distribution. The median TTM/ZTM/FM multiplicities are 44/22/36, respectively. The right plot of Fig. 2 shows the FM distribution for all of the models described in Sect. 2.1.

\section{Results}

\subsection{Strangeness enhancement}

We present here some strangeness (and baryon) enhancement variables, considering the multiplicities of strange hadrons produced inside and outside the leading jet. Figure 3 shows the ratio of various strange hadron yields as a function of the FM criteria (TTM and ZTM are in Appendix B). The left (right) panels represent the case of default (Rope) 

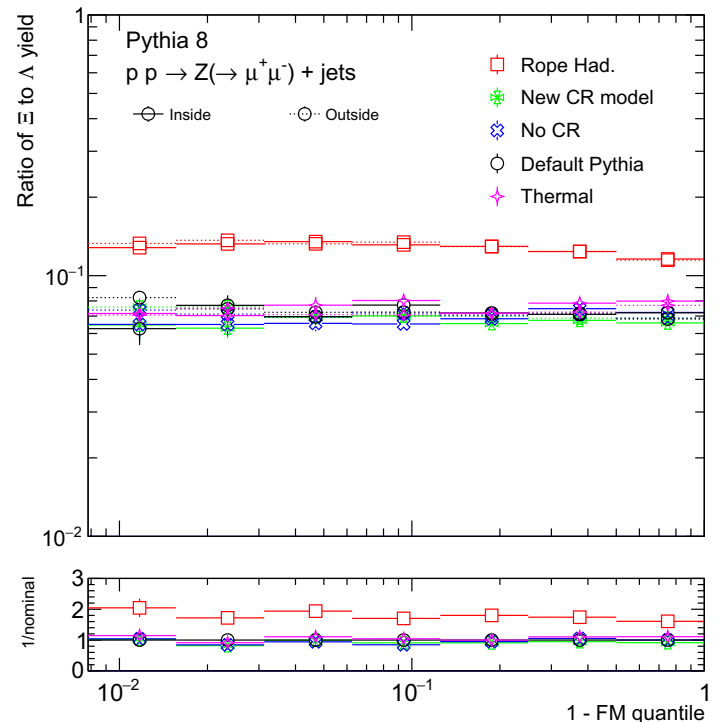

Fig. 5 Strangeness (left) and baryon (right) enhancement using the $\Xi$ to- $\Lambda$ (left) and $\Lambda$-to-Kaon (right) ratios as a function of the FM quantile for the various models described in Sect. 2.1 outside of jets. Low values

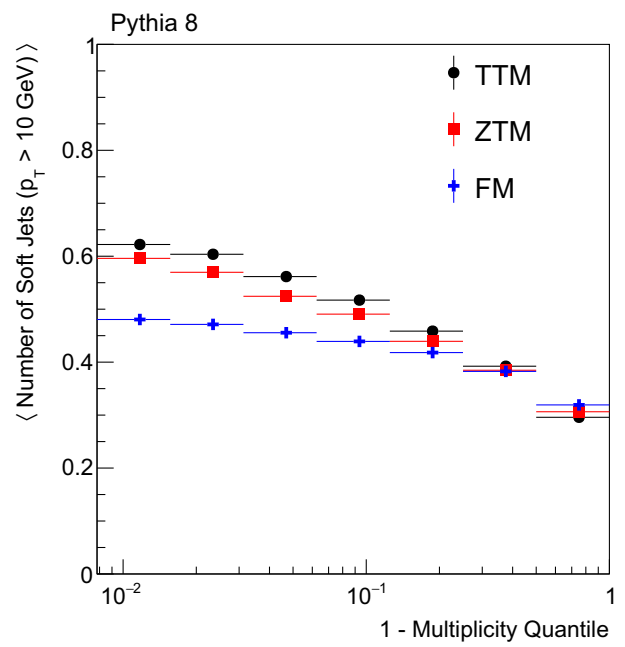

Fig. 6 Left: The number of soft $\left(10 \mathrm{GeV}<p_{\mathrm{T}, J}<20 \mathrm{GeV}\right)$ jets as a function of the multiplicity quantile for the three multiplicity definitions. Right: the gluon composition of the hard jet as a function of the multiplicity quantile. There is no unique way to define the quark or gluon

Pythia hadronization. For all definitions of underlying track multiplicity we notice similar behaviors: no evidence of strangeness enhancement in the case of pure Pythia, compared to the expected clear enhancement in the case of Rope fragmentation. We note the overall increase of strange production in the case of Rope fragmentation with respect to Pythia, independently of the track multiplicity.

In order to study strangeness and baryon enhancement inside jets, it is useful to subtract the contribution from the underlying event (UE) using an areas-based approach as
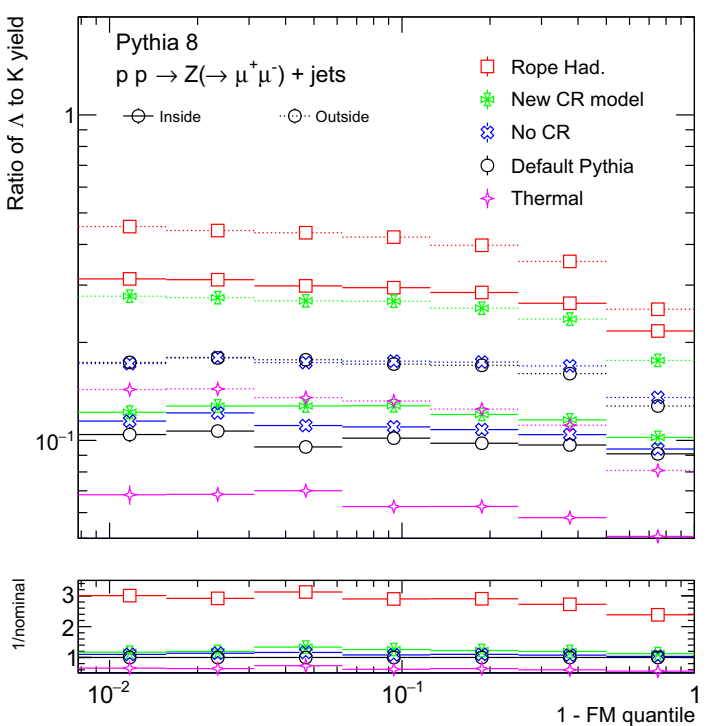

of the $x$-axis correspond to extreme multiplicities; the rightmost bin captures events with multiplicities at or below the median FM

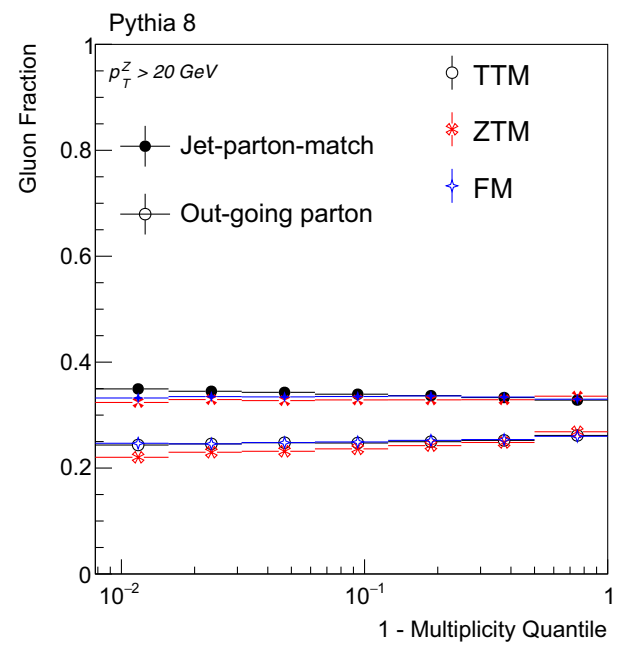

nature of a jet; we show two different definitions: jet-parton-match is the type of the highest energy parton found within the $\Delta R<0.4$ of the jet axis and out-going parton is the type of the out-going parton from the matrix element. Higher multiplicities are to the left

described in Sect. 2.2. The following corrected multiplicity and momentum ratios are defined:

$\frac{n_{\mathrm{cor}}^{h_{1}}}{n_{\mathrm{cor}}^{h_{2}}}=\frac{n^{h_{1}}-\rho_{\#}^{h_{1}} \times A}{n_{\mathrm{cor}}^{h_{2}}-\rho_{\#}^{h_{2}} \times A}$,

where $h_{i}$ are hadron species, $\rho_{\#}$ is the background number density and $A$ is the jet area. Similarly, the corrected momen- 


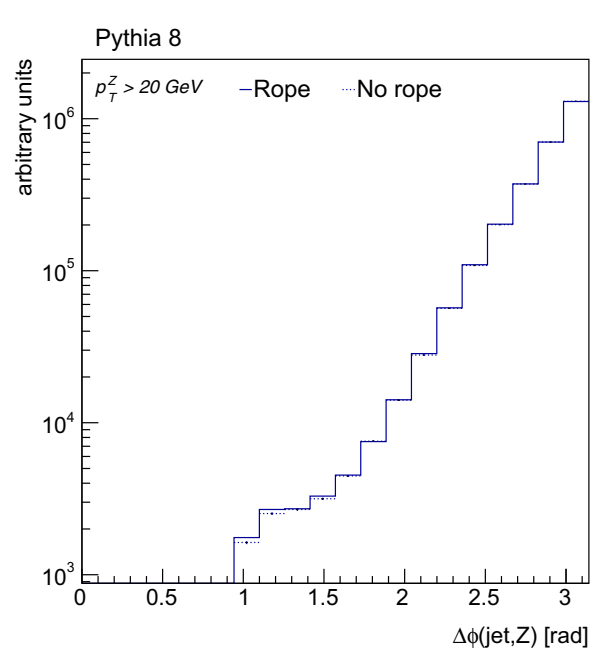

Fig. 7 Left: The azimuthal angle between the hard jet and the $Z$ boson. Most events are nearly back-to-back; events are vetoed in the region where there is a strong additional recoil (even if it does not form a jet

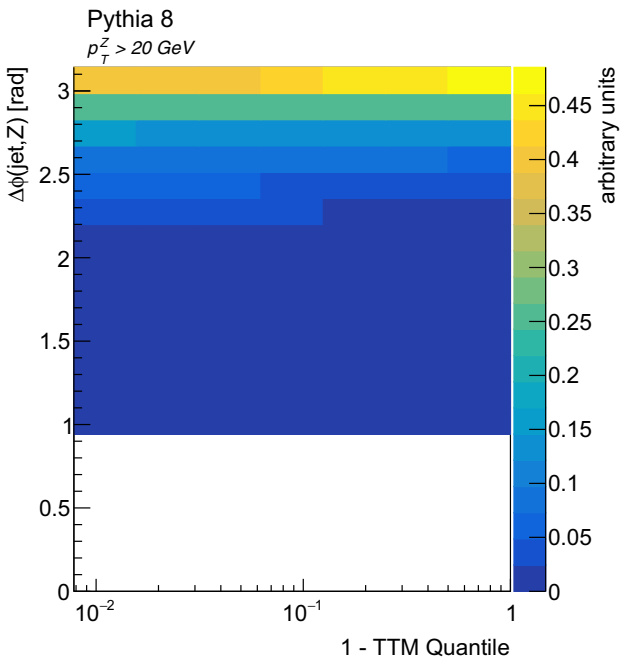

above threshold). Right: the multiplicity dependence of the azimuthal angle distribution. There is a slight broadening at higher multiplicities (on the left); this is not present for FM
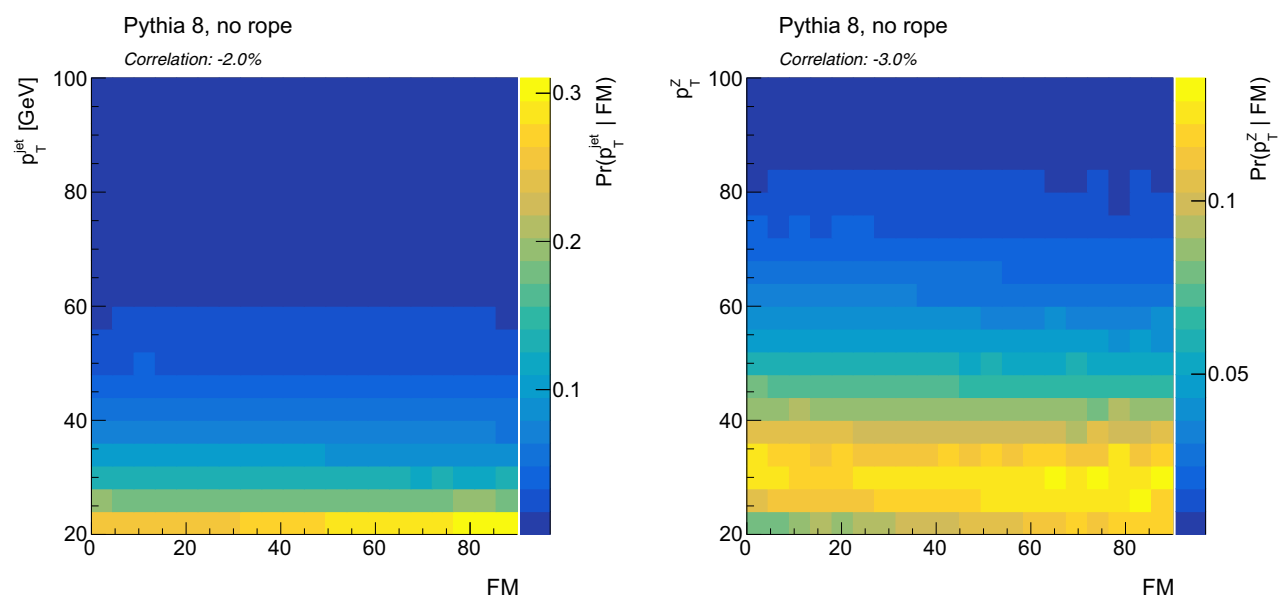

Fig. 8 The distribution of the jet (left) or $Z$ boson (right) $p_{\mathrm{T}}$ given the event multiplicity defined by FM. The linear correlation coefficient is presented at the top of each plot. The corresponding plots for TTM and ZTM are shown in the appendix, Fig. 17

tum fraction carried by identified hadrons is given by

$z^{\text {corr }}=\frac{p_{\mathrm{T}}^{h_{1}}-\rho^{h_{1}} \times A}{p_{\mathrm{T}, \mathrm{jet}}-\rho \times A}$

where $\rho^{h_{i}}$ is the background momentum density for hadron species $h_{i}$ (and $\rho$ is the standard momentum density). There is some freedom in defining $\rho_{\#}$ and $\rho$; we have tested both the standard median approach and one based on the mean where the selected jet is removed. The jets used are the same as for the standard approach - see Sect. 2.2. Since the number and momentum carried by identified hadrons can be very small (often zero), the median is not a good estimator of the contribution. Therefore, we use the mean.
Figure 3 shows that the ratio of strange hadron fractions inside and outside the jet remains rather constant, for all track multiplicity definitions, for all quantile values, and is also approximately the same for the Rope vs Pythia fragmentations. These trends are also nearly the same for the multiplicity and the average momentum fraction carried by the hadrons (Fig. 4). A subset of the comparisons from Fig. 3 focusing on decoupling strangeness enhancement and baryon enhancement are shown in Fig. 5 for all the models discussed in Sect. 2.1. The Rope hadronization, thermal hadronization, and new CR models all show signs of strangeness enhancement, with the biggest effect from the rope hadronization. All of the models predict baryon enhancement at high multiplicity, with the rope and new CR models predicting an increase on top of the default trend. The thermal model does show an 


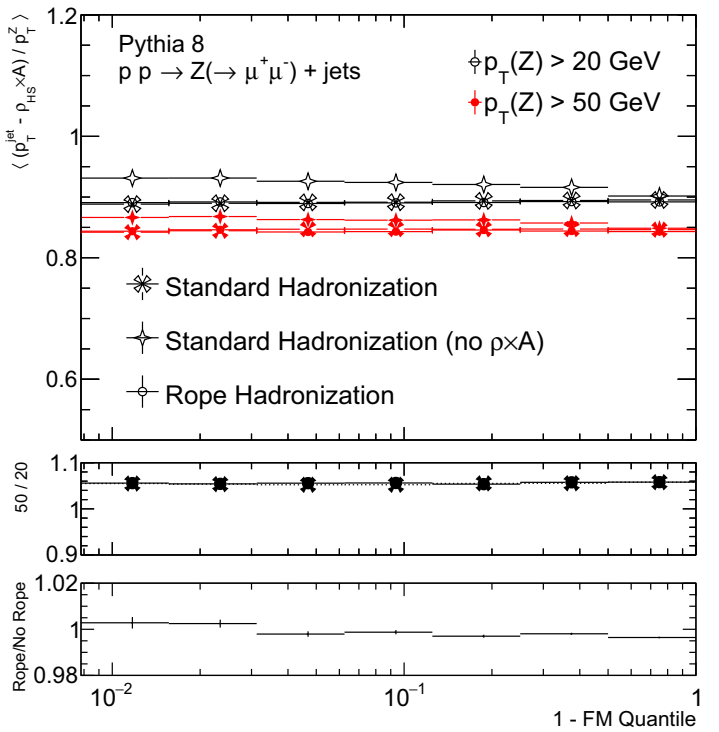

Fig. 9 The average fractional transverse momentum imbalance between leading jet and $Z$ boson, $x_{Z J}=p_{\mathrm{T}, J} / p_{\mathrm{T}, Z}$, as a function of track multiplicity quantile, FM. Three curves in the upper panels indicate the values for the standard hadronization with and without an area correction as well as the rope hadronization with the correction. Higher multiplicities are to the left. The middle panel shows the ratio of the higher to lower $Z$ boson $p_{\mathrm{T}}$ cut (standard hadronization is shown

excess increase over the default model, but the low multiplicity baryon yield is below the default one. The decomposed ratios suggest that while the strangeness enhancement is predicted to be about the same inside and outside of jets for the Rope hadronization model, the baryon enhancement is much bigger outside of jets. ${ }^{4}$ Results from ALICE suggest that all of the enhancement inside jets is due to the UE [102], with no additional modification from the jet itself. ${ }^{5}$ The Rope hadronization does indicate additional Baryon enhancement beyond the UE contribution, albeit at a reduced level. This is also true for the other models shown in the right plot of Fig. 5.

\subsection{Jet balancing}

We study in this section a typical observable associated with the presence of a quark-gluon plasma, namely the jet energy loss, leading to an imbalance in the transverse momentum

\footnotetext{
4 This may seem to be a contradiction with Fig. 3, but since the corrections in Eq. 1 are applied jet-by-jet, the ratio of the $\Lambda$ to pion line to the $K$ to pion line in Fig. 3 is not equivalent to Fig. 5. It is therefore important to study multiple pairs of ratios to expose the full behavior.

5 We thank our referee for pointing out that any additional enhancement predicted by our implementation of the Rope model could be a feature of the setup. Ropes are formed regardless of how fast the strings are moving away from the point of interaction. This may be inadequate inside jet regions where strings can travel a significant distance prior to hadronizing.
}

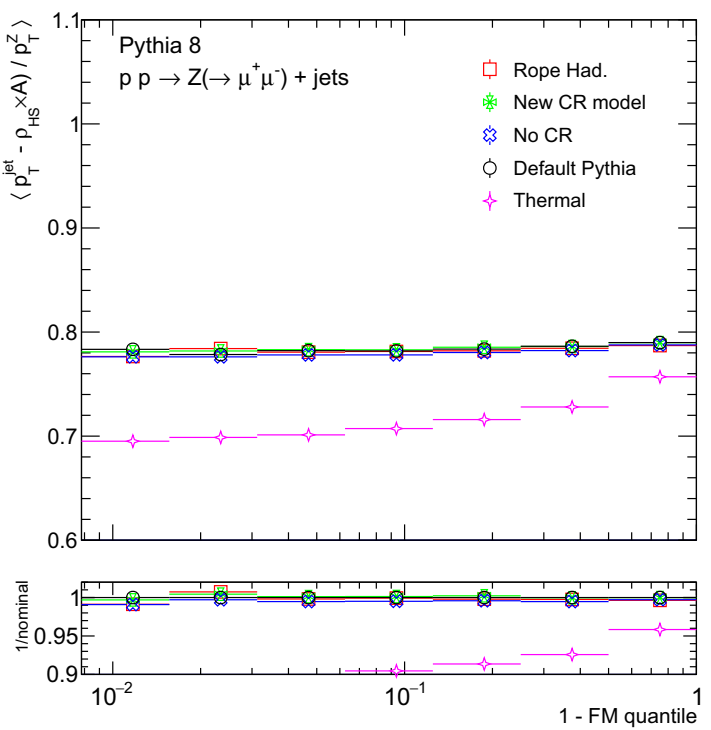

with a dashed line, rope with solid), both including the areas correction. Finally, the lower panel shows the ratio between the rope and standard hadronization models with the $p_{\mathrm{T}, Z}>20 \mathrm{GeV}$ requirement and both with the areas correction. The corresponding plots for TTM and ZTM are in the appendix, Fig. 18. The right plot shows similar information for the single $p_{\mathrm{T}}(Z)>20 \mathrm{GeV}$ threshold and multiple models

between a jet and its recoil. The cleanest final state in which such phenomenon can be exposed is the recoil of a jet against an electroweak gauge boson, which does not interact with the possible plasma. In particular, we focus on the case of a $Z$ boson decaying to leptons, whose momentum can be well measured, and whose identification is largely free of backgrounds. The study of the $Z$-jet balance at large transverse momentum, as a function of track multiplicity, requires however some caution, since radiation from the hard process will influence the momentum balance, and at the same time it will sculpt the underlying track multiplicity. A different track multiplicity could also reflect a different composition of the initial and final states $(q \bar{q} \rightarrow g Z$ vs $q g \rightarrow q Z)$. All these effects might in principle induce an imbalance that emulates a quenching trend at the highest track multiplicities. The extent of such correlations between track multiplicity, hard radiation and initial state composition is shown in Fig. 6. The left plot shows the average multiplicity of soft $\left(p_{\mathrm{T}, J}>10 \mathrm{GeV}\right)$ jets versus the multiplicity quantile, showing that high multiplicity events (lower quantiles) are correlated to a larger radiation activity. As expected, the correlation is strongest for TTM, then ZTM, and weakest for FM. This will influence the ratio of the $Z$ boson $p_{\mathrm{T}}$ to the jet $p_{\mathrm{T}}$ and can also be observed to broaden the distribution of the angular separation, as in Fig. 7. The right plot of Fig. 6 shows on the other hand a minor, if any, dependence of the gluon final-state fraction versus track multiplicity. Similarly, there is little correlation 


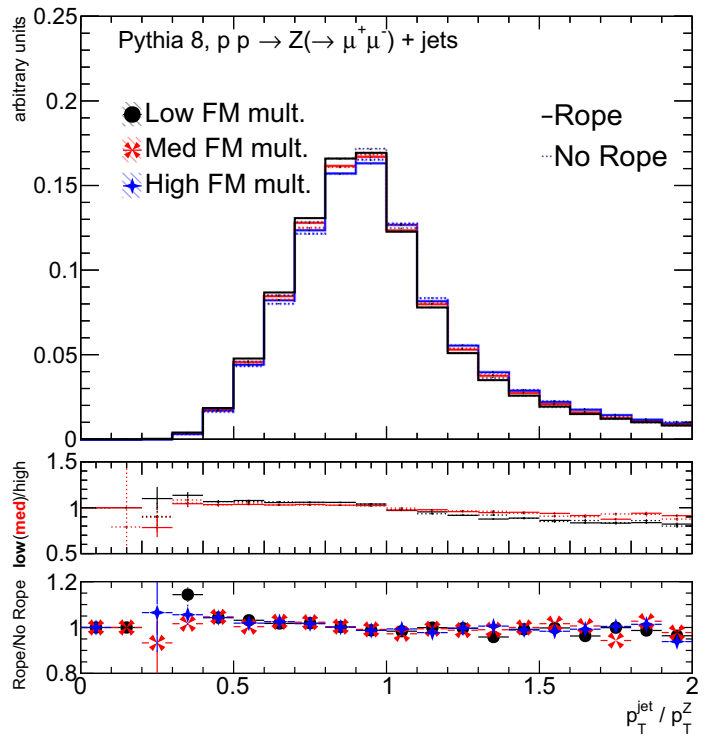

Fig. 10 The distribution of the ratio $x_{Z J}=p_{\mathrm{T}, J} / p_{\mathrm{T}, Z}$ for three bins of the TTM (top left), ZTM (top right), or FM (bottom) multiplicity: the 50th, 75th, and 90th percentile. The middle panel shows the ratio of the distribution for high to low multiplicity for both hadronization models (standard hadronization with a dotted line). In the lower panel, the ratio between the Rope and standard hadronization models is displayed for all three multiplicity regions. The corresponding plots for TTM and ZTM are in the appendix, Fig. 19

between the jet or $Z p_{\mathrm{T}}$ itself on the event multiplicity, as shown in Fig. 8.

With these observations in mind, we show in Fig. 9 the average fractional transverse momentum imbalance between leading jet and $Z$ boson, $x_{Z J}=p_{\mathrm{T}, J} / p_{\mathrm{T}, Z}$, as a function of track multiplicity quantile, focusing on the least biased multiplicity indicator FM. Results are shown for two thresholds of $p_{\mathrm{T}, Z}>20$ and $>50 \mathrm{GeV}$, considering the cases of Pythia and Rope fragmentation. Except where indicated by the caption "no $\rho \times \mathrm{A}$ ", an average subtraction of the underlying event activity inside the jet cone is performed. The distributions with respect to the FM quantile are independent of multiplicity, and appear not to be influenced by a possible hard radiation bias that exists for the TTM and ZTM. This suggests that FM would be a robust variable to explore the possible presence of quenching-induced imbalance. The right plot of Fig. 9 shows that nearly all of the models predict the same trend with multiplicity except the thermal model, which exhibits extreme 'quenching'-like behavior at high multiplicity.

Figure 10 shows the full distribution of the ratio in three bins of multiplicity. Nearly independent of the percentile, the standard deviation of the ratio distribution is about $20 \%$. With about 500 jets in the $1 \%$ percentile category (Fig. 1), the statistical precision in the determination of $x_{Z J}$ will be $\lesssim 1 \%$. The experimental resolution should be comparably small. The high multiplicity single $p p$ collisions have comparable multiplicity to low/moderate pileup bunch crossings, similar to the levels with early Run 2 . Therefore, one can estimate the uncertainty in the reconstructed jet energy due to pileup as an estimate of the uncertainty for high multiplicity single $p p$ collisions [103,104]. As a figure of merit, a $1 \mathrm{GeV}$ energy loss would correspond, for a $20 \mathrm{GeV}$ jet, to a $5 \%$ effect on $\left\langle x_{Z J}\right\rangle$.

\subsection{Jet substructure}

In addition to reducing the total energy inside a jet, interactions with the QGP in HI collisions distort the radiation pattern. Scattering with the medium results in jets with a broader distribution of energy and therefore jet substructure tools may be used to search for a QGP in central $p p$ collisions. The soft drop jet grooming procedure [105] (the generalization of modified mass drop [106] when $\beta \neq 0$ ) has gained a lot of recent attention theoretically and experimentally because of its insensitivity to non-global logarithms and robustness to wide angle and soft radiation. Therefore, soft drop jet observables are useful for probing if the structure of a jet has changed in events with high multiplicity. In addition to the jet mass, another important soft drop jet observables is the fraction of the groomed jet's momentum carried by the subleading subjet, $z_{g}$. This observable has the interesting property that it is independent of $\alpha_{s}$ at leading order and is directly related to the QCD splitting functions in vacuum [107]. Therefore, any change in the $z_{g}$ distribution in high multiplicity events may be an indication of a modification of the partonic fragmentation function. Preliminary results from CMS are suggestive of medium-induced modifications of the $z_{g}$ distribution [108], which are also predicted by various models of jet quenching [109-113], although there may $\mathrm{be}^{6} \mathrm{a}$ tension with preliminary STAR results [114]. Figure 11 shows the distribution of the soft drop mass and $z_{g}$ as a function of FM with the standard and Rope hadronization models using $z_{\text {cut }}=0.1$ and $\beta=0$. The mass does show a dependence on the multiplicity, which may be due in part to a residual contribution within the catchment area of the subjets not removed from grooming. There is also a significant difference in the shape of the mass distribution between the two hadronization models, though there is little dependence of the difference on multiplicity. The multiplicity dependence is much reduced in the case of the $z_{g}$ spectra, and in particular the FM dependence is particularly flat (TTM and ZTM are in the appendix), confirming the smaller radiation bias of the FM distributions. As for the mass distributions, the $z_{g}$ spectra are different in the case of Pythia and Rope fragmentation, but the difference is not affected by the multiplicity.

\footnotetext{
6 We say 'may be' because the analyses are not identical so differences may arise from the approach.
} 


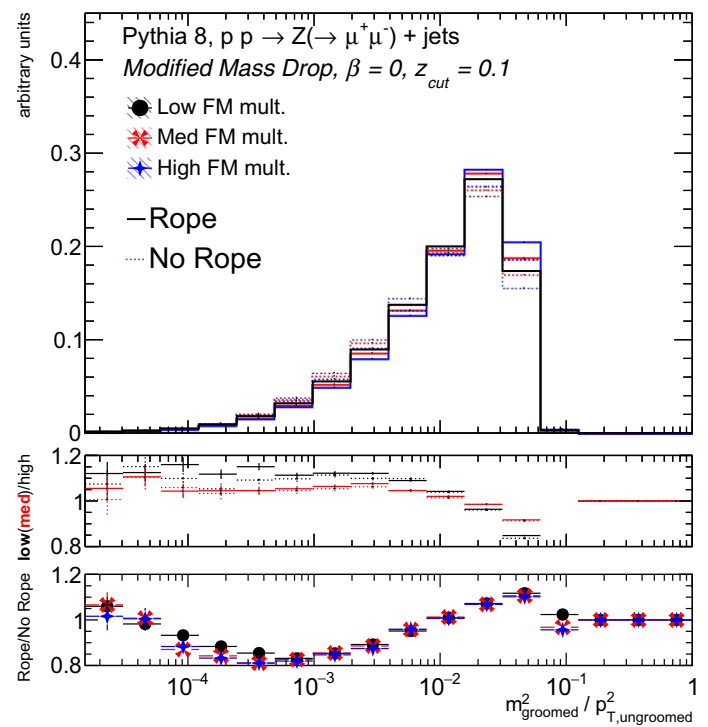

Fig. 11 Modified Mass Drop (also Soft Drop with $\beta=0$ ) jet mass (left) and momentum sharing $z_{g}$ (right) for three bins in FM that correspond to the 50th, 75, and 90th percentiles. The middle panel shows the ratio of the distributions for high to low multiplicity for both hadronization models (standard hadronization with a dotted line). In the lower

However, there is a small difference in the impact of the Rope hadronization on the effect of high multiplicity for the $z_{g}$ distribution. The Rope hadronization predicts a lower multiplicity (Fig. 2) and thus more energy per particle. This results in more jets that are completely removed from grooming (less pronounced second subjet). However, given that there is a second subjet, the energy sharing is more equal as the energy is confined to fewer hadrons (thus they have higher energy per hadron). Fitting the lower ratio panel of the FM $z_{g}$ distribution to a polynomial, ${ }^{7}$ as shown in Fig. 12, results in a constant term that significantly differs within the MC statistics, which are about $30 \%$ higher than the $7 \mathrm{TeV}$ data statistics (the linear and quadratic terms also differ, but not as significantly). The size of the effect is about $10 \%$.

The additional models introduced in Sect. 2.1 are shown in Fig. 13. The thermal model shows similar trends to the Rope hadronization for the mass, but the shift in the $z_{g}$ distribution is less pronounced than the Rope model. The new CR model predicts little impact on the mass and $z_{g}$.

Another well-studied jet substructure observable is the fraction of a jet's momentum carried by identified particles. Figure 14 shows this variant of the fragmentation function in various regions of TTM with and without the Rope hadronization model. For both models, a higher multiplicity corresponds to a softer spectrum. This is due in part to the increased multiplicity of UE that happens to fall in

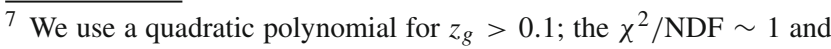
does not significantly improve for higher order polynomials.
}

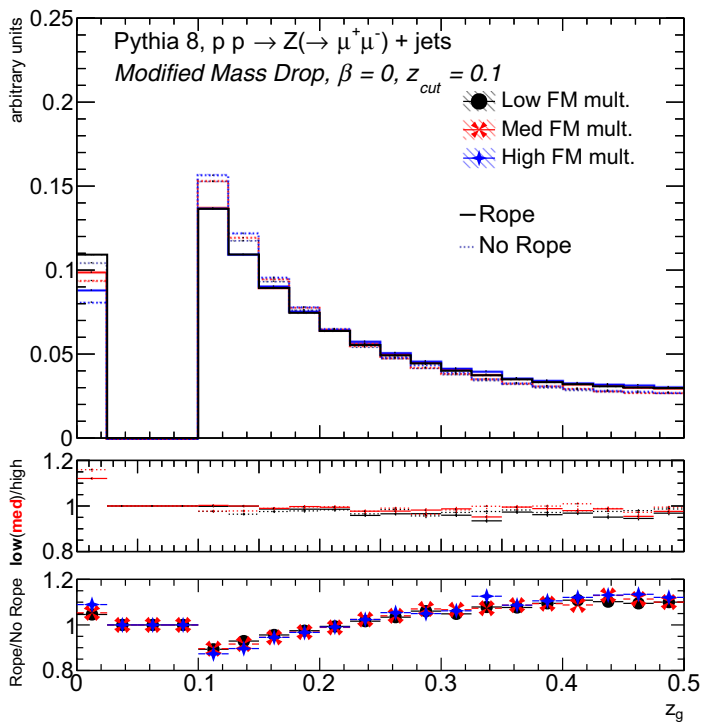

panel, the ratio between the Rope and standard hadronization models is displayed for all three multiplicity regions. Due to the algorithm value $z_{\text {cut }}=0.1, z_{g} \geq 0.1$; when the entire jet is groomed away, $z_{g}=0$. The corresponding plots for TTM and ZTM are in the appendix, Fig. 20

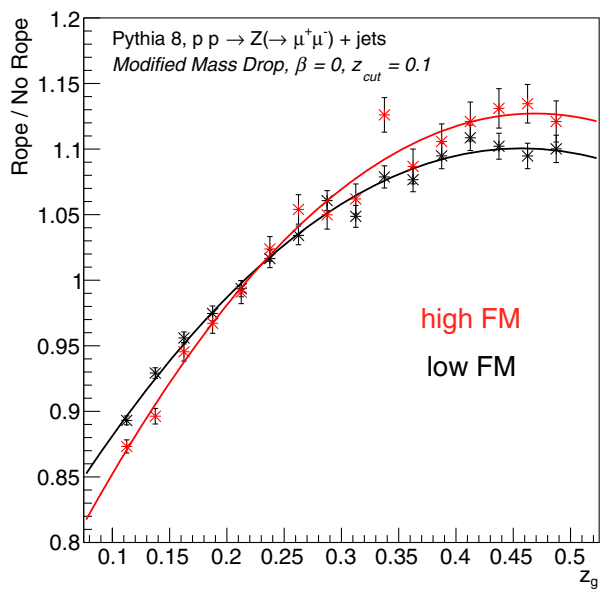

Fig. 12 The quadratic fits to the low- and high-multiplicity Rope/No Rope $z_{g}$ spectra shown for FM in Fig. 11

the jet catchment area. Interestingly, there is a multiplicitydependent difference in this effect between the Rope and standard hadronization models for pions, though it is less clear for kaons (due in part to limited MC statistics).

\section{Conclusions and future outlook}

Proton-proton interactions can be much more complex than vacuum parton-parton interactions. When collided with significant overlap, collective effects observed also in more 

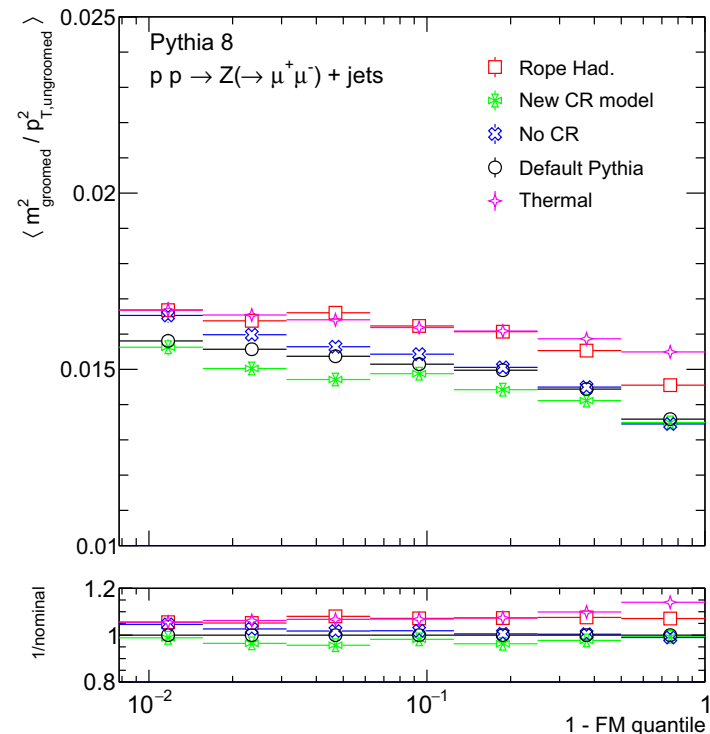

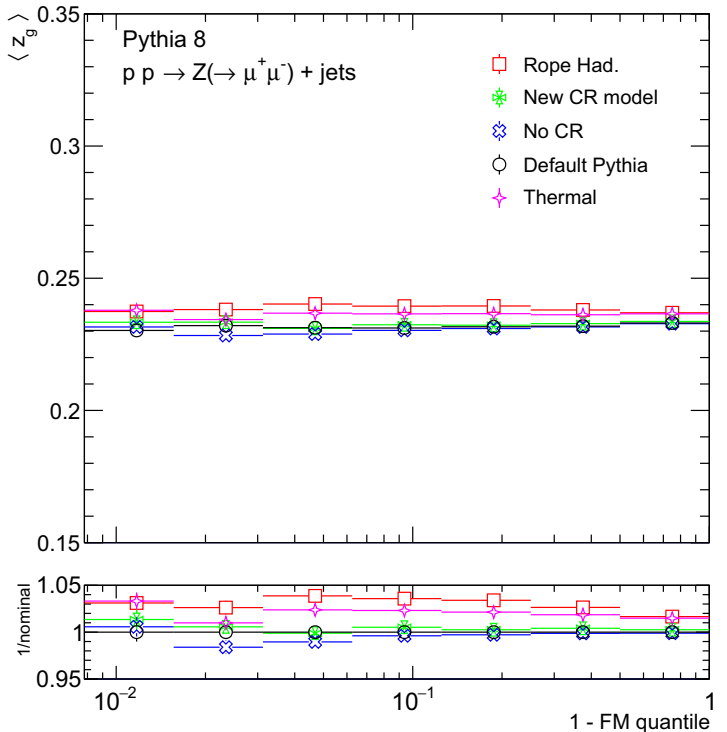

Fig. 13 The average mass (left) and $z_{g}$ (right) as a function of the FM multiplicity and various models of collective effects. Higher multiplicities are to the left

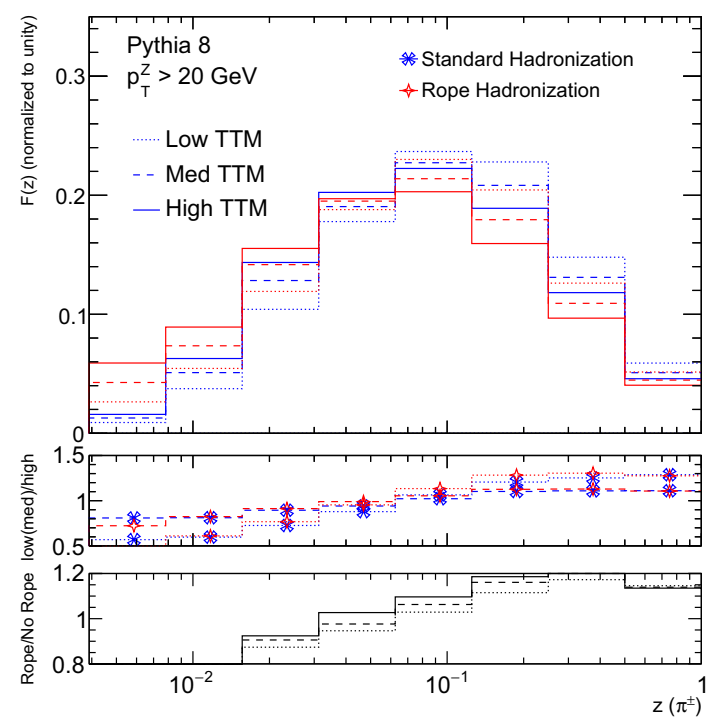

Fig. 14 The distribution of the momentum fraction $(z)$ carried by pions (left) and kaons (right) for low $(<50)$, medium $(50<$ TTM $<100)$, and high $(>100)$ TTM for both the default and Rope hadronization models. The middle panel shows the ratio of the distributions for high

extended systems are suggestive of a common origin. Using MC simulations of models with collective effects, we have studied observables related to jets that may be sensitive to the source of the observed phenomena. No one model can explain all of the observed collective effects, but they provide useful benchmarks for probing quenching-like behavior. Interpreting the strangeness enhancement in high multiplicity $p p$ as a sign of a QGP, it will be interesting to next try to quantify the expected size of such a QGP by scaling up to HI and then predicting the magnitude of potential jet quenching. We note that several studies have appeared in the literature, discussing the possibility of a QGP formation in $p p$ and $\mathrm{pA}$

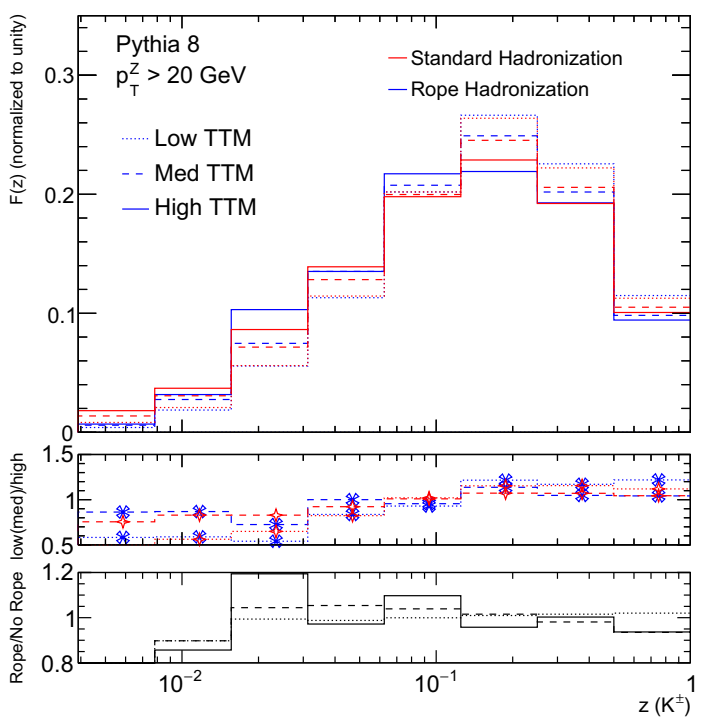

to low multiplicity for both hadronization models. In the lower panel, the ratio between the Rope and standard hadronization models is displayed for all three multiplicity regions

collisions [115-118]. Their conclusions and estimates of the properties of this QGP state vary significantly, and we are not in the position to project in a robust way their impact on the jet quenching effects that could arise in the context of the $Z+$ jet observables discussed here. This would require setting up an event simulation framework incorporating the possible development of a QGP in $p p$ collisions, a task that goes beyond the scope of our simple study, and which will hopefully be picked up by more expert colleagues.

Independently of the possible quenching effects induced by a mini-QGP, our study shows some interesting features of the Rope fragmentation, and differences with respect to the 
standard Pythia fragmentation at the level of several percent, when considering jet-related quantities, such as the groomed mass and the $z_{g}$ spectra. The experimental study of these quantities can therefore provide additional handles in the tuning of these alternative fragmentation models, or in the development of new ones. Measurements with $Z / \gamma+$ jets should be possible with high precision using ATLAS and CMS and the observables and trends presented here provide a baseline for a full experimental investigation.

Acknowledgements We would like to thank the organizers and participants of the "Collective effects in small collisions systems" workshop at CERN for stimulating discussions. In addition, we are grateful to Jesse Thaler and Peter Jacobs for useful discussions and for recommending the study of jet substructure observables and forward multiplicity, respectively. This work was supported in part by the Office of
High Energy Physics of the U.S. Department of Energy under contract DE-AC02-05CH11231.

Open Access This article is distributed under the terms of the Creative Commons Attribution 4.0 International License (http://creativecomm ons.org/licenses/by/4.0/), which permits unrestricted use, distribution, and reproduction in any medium, provided you give appropriate credit to the original author(s) and the source, provide a link to the Creative Commons license, and indicate if changes were made.

Funded by SCOAP ${ }^{3}$.

\section{A Parameter settings}

Table 1 describes all of the simulation parameters used in this paper.

Table 1 The parameters used for the various models described in this paper. A '-' indicates that the same value as the default model is used

\begin{tabular}{|c|c|c|c|c|c|}
\hline Parameter & Default Pythia & Rope & New CR & No CR & $\begin{array}{r}\text { Thermal } \\
\text { Model }\end{array}$ \\
\hline StringPT: sigma & 0.335 & 0.31 & - & - & - \\
\hline Stringz:aLund & 0.68 & 0.38 & 0.36 & - & - \\
\hline Stringz: bLund & 0.98 & 0.37 & 0.56 & - & - \\
\hline StringFlav: probstoud & 0.19 & 0.21 & 0.2 & - & - \\
\hline StringFlav:probSQtoQQ & 1.0 & 0.915 & - & - & - \\
\hline StringFlav: probQQ1 toQQ 0 & 0.027 & 0.0275 & - & - & - \\
\hline StringFlav: probQQ1 toQQ0join & $0.5,0.7,0.9,1.0$ & - & $0.0275,0.0275,0.0275,0.0275$ & - & - \\
\hline StringFlav: probedtod & 0.09 & 0.073 & 0.078 & - & - \\
\hline Stringz:aExtraDiquark & 0.5 & 0.97 & - & - & - \\
\hline RadiusRatio & N/A & 0.2 & - & - & - \\
\hline Rapidityspan & N/A & 0.5 & - & - & - \\
\hline MultiPartonInteractions:pToRef & 2.15 & - & - & - & 2.5 \\
\hline BeamRemnants: remnantMode & 0 & - & 1 & - & - \\
\hline ColourReconnection: reconnect & On & - & - & Off & - \\
\hline ColourReconnection:mode & 0 & - & 1 & - & - \\
\hline ColourReconnection:range & 1.8 & - & - & - & 1.1 \\
\hline ColourReconnection:allowDoubleJunRem & On & - & Off & - & - \\
\hline ColourReconnection:m0 & N/A & - & 0.3 & - & - \\
\hline ColourReconnection:allowJunctions & N/A & - & On & - & - \\
\hline ColourReconnection: junctionCorrection & N/A & - & 1.2 & - & - \\
\hline ColourReconnection:timeDilationMode & N/A & - & 2 & - & - \\
\hline ColourReconnection:timeDilationPar & $\mathrm{N} / \mathrm{A}$ & - & 0.18 & - & - \\
\hline StringPT: thermalModel & Off & - & - & - & On \\
\hline StringPT: temperature & N/A & - & - & - & 0.21 \\
\hline StringFlav: BtoMratio & N/A & - & - & - & 0.357 \\
\hline StringFlav: StrangeSuppression & N/A & - & - & - & 0.5 \\
\hline StringPT: expNSP & N/A & - & - & - & 0.13 \\
\hline HadronLevel: HadronScatter & Off & - & - & - & On \\
\hline HadronScatter:mode & N/A & - & - & - & 0 \\
\hline HadronScatter:maxProbds & N/A & - & - & - & 0.5 \\
\hline Reference: & [94] & {$[88]$} & {$[93]$} & & {$[14]$} \\
\hline
\end{tabular}




\section{B Plots with alternative multiplicity definitions}

Figures 15 and 16 show plots of strangeness enhancement Pfor the TTM and ZTM multiplicity definitions (Figs. 17, $18,19,20)$.

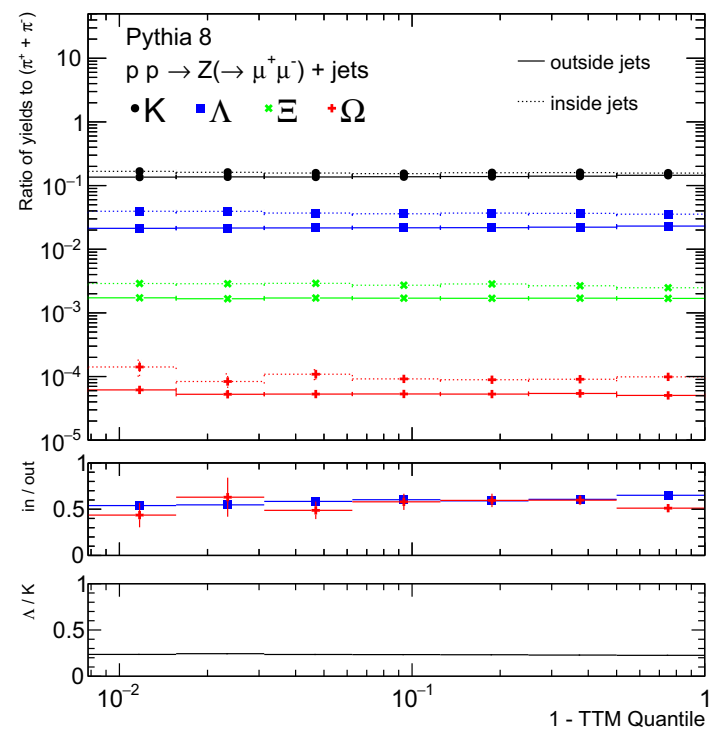

Fig. 15 Same as Fig. 3, but as a function of the TTM quantile

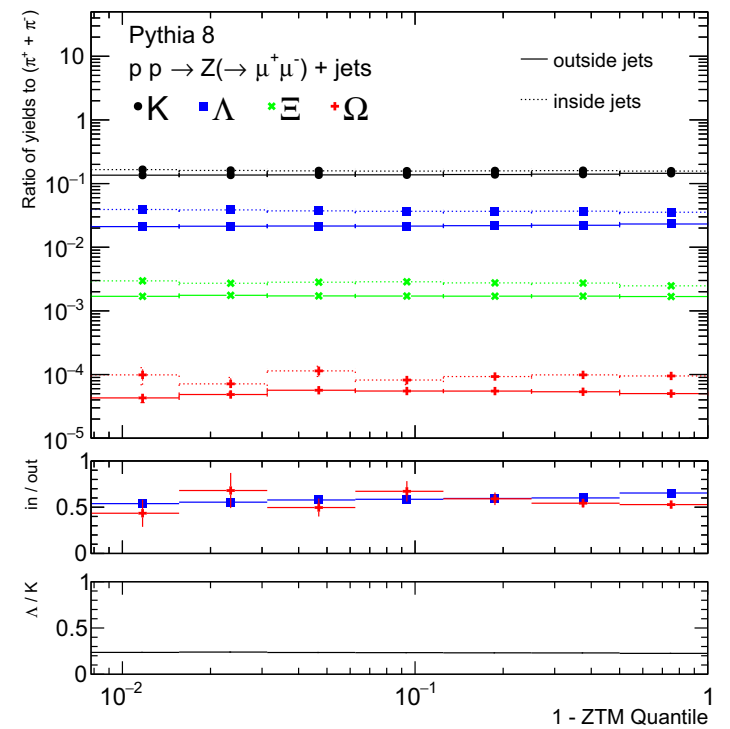

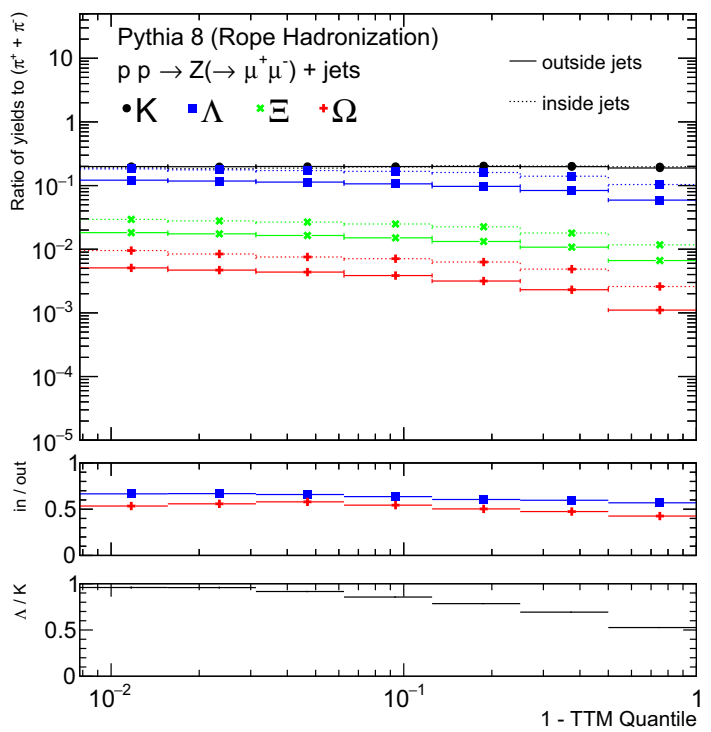

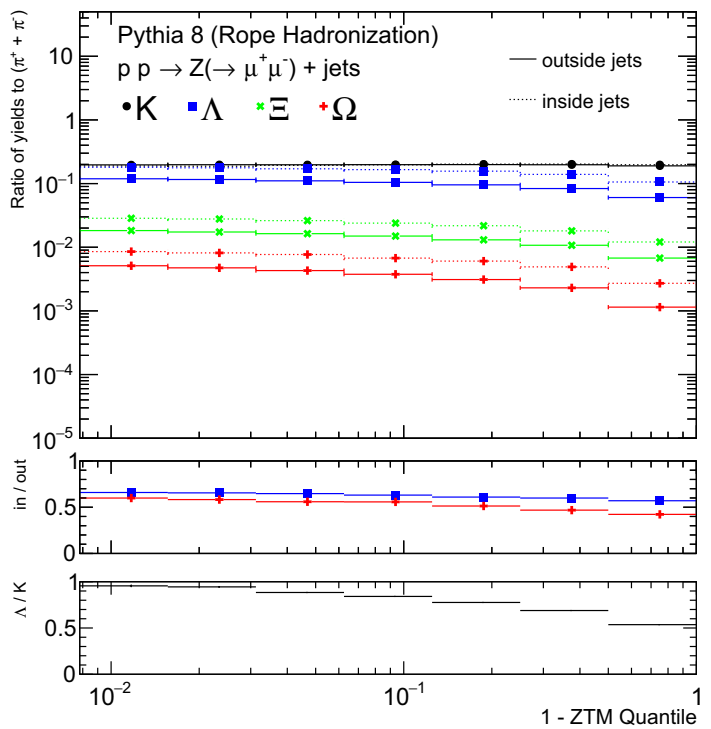

Fig. 16 Same as Fig. 3, but as a function of the ZTM quantile 

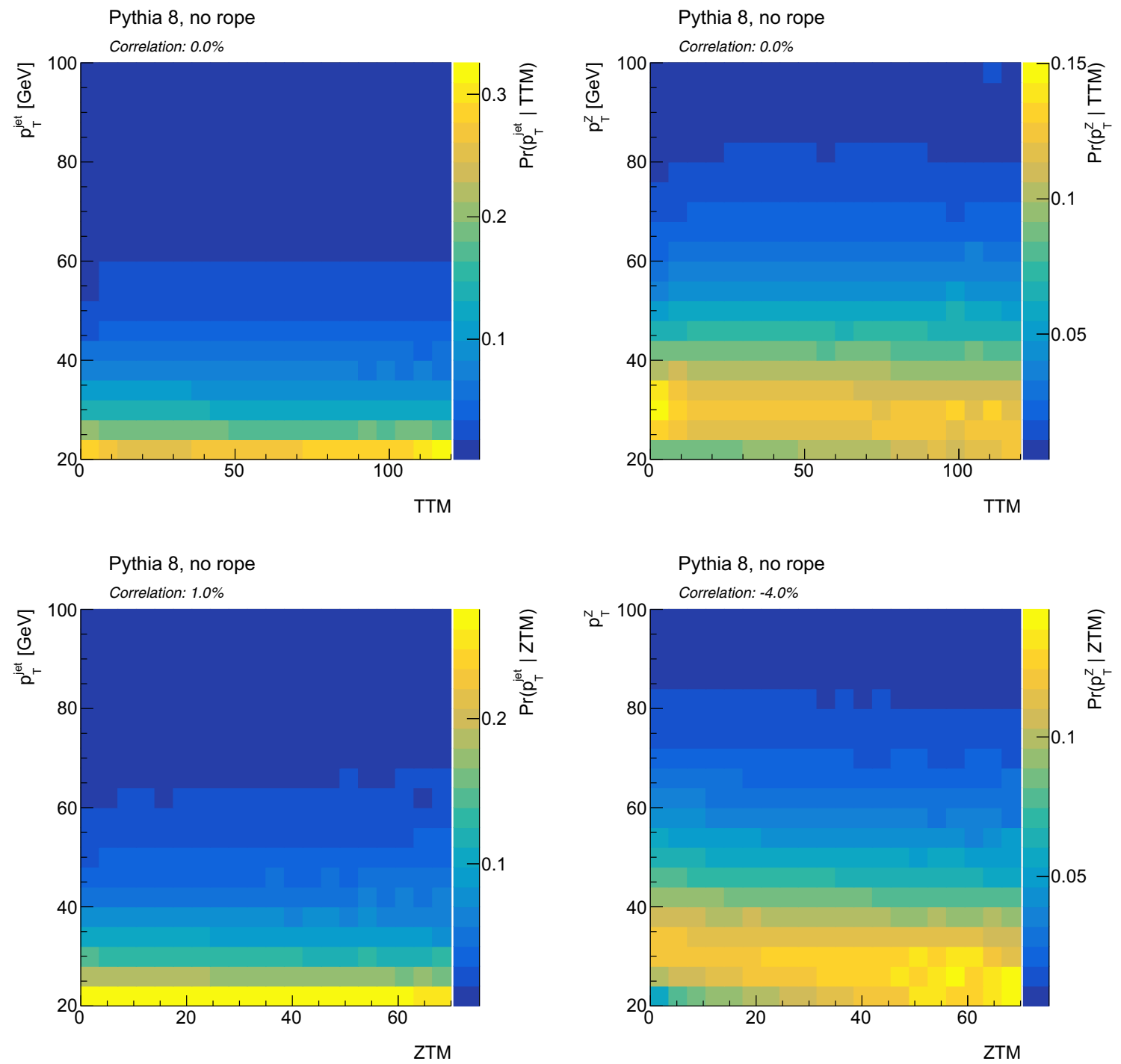

Fig. 17 The distribution of the jet (left) or $Z$ boson (right) $p_{\mathrm{T}}$ given the event multiplicity defined by TTM (top) and ZTM (bottom). The linear correlation coefficient is presented at the top of each plot 
Fig. 18 The average fractional transverse momentum imbalance between leading jet and $Z$ boson, $x_{Z J}=p_{\mathrm{T}, J} / p_{\mathrm{T}, Z}$, as a function of track multiplicity quantile, for TTM (left) and ZTM (right). Three curves in the upper panels indicate the values for the standard hadronization with and without an area correction as well as the rope hadronization with the correction. Higher multiplicities are to the left. The middle panel shows the ratio of the higher to lower $Z$ boson $p_{\mathrm{T}}$ cut (standard hadronization is shown with a dashed line, rope with solid), both including the areas correction. Finally, the lower panel shows the ratio between the rope and standard hadronization models with the $p_{\mathrm{T}, Z}>20 \mathrm{GeV}$ requirement and both with the areas correction
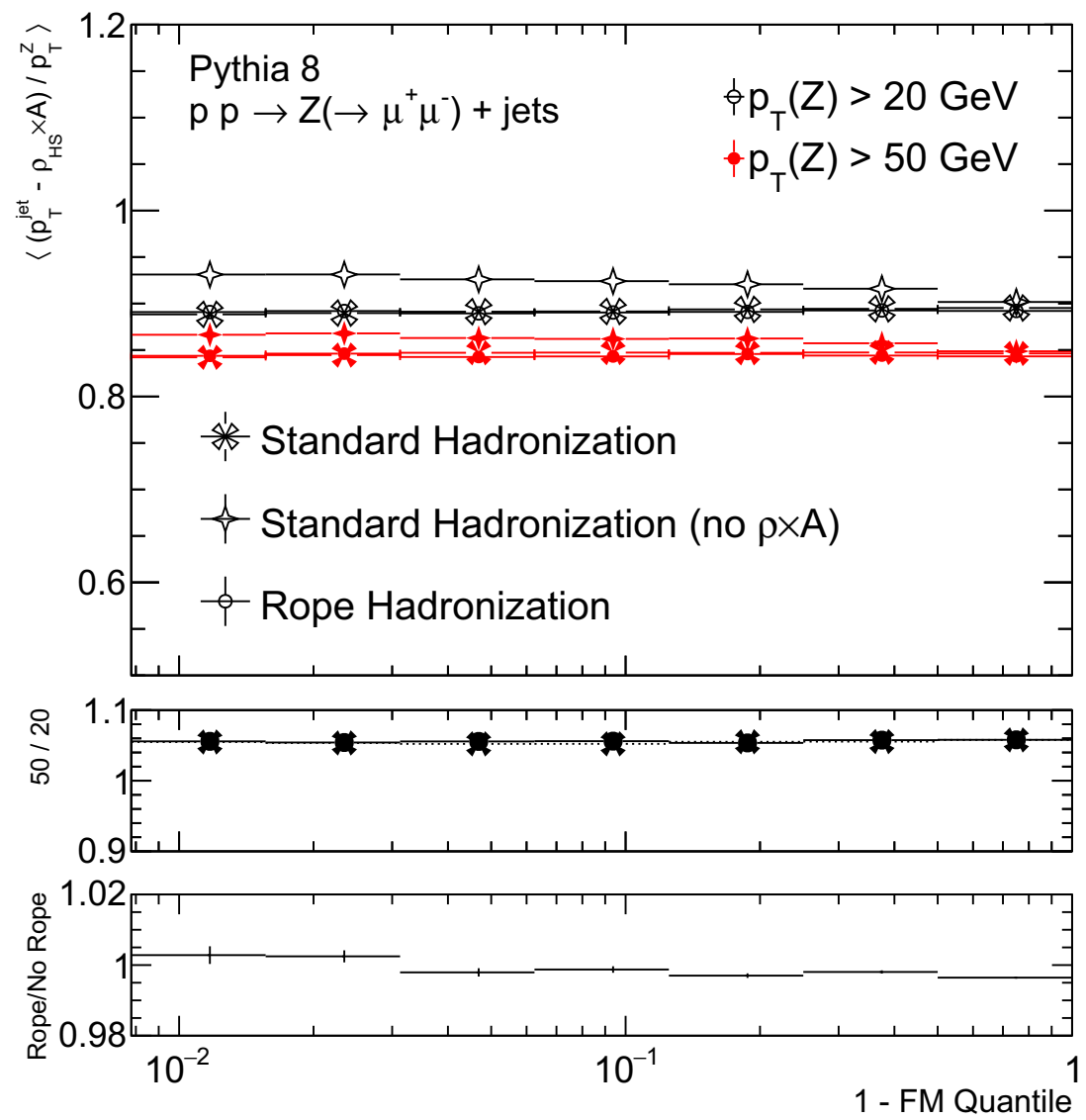

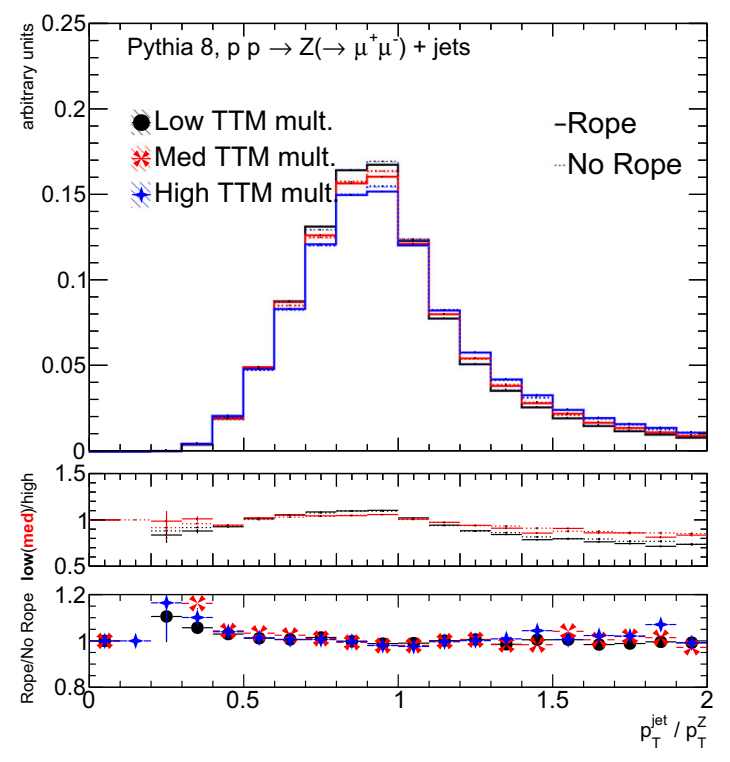

Fig. 19 The distribution of the ratio $x_{Z J}=p_{\mathrm{T}, J} / p_{\mathrm{T}, Z}$ for three bins of the TTM (left) and ZTM (right) multiplicity: the 50th, 75th, and 90th percentile. The middle panel shows the ratio of the distribution for high to low multiplicity for both hadronization models (standard hadroniza-

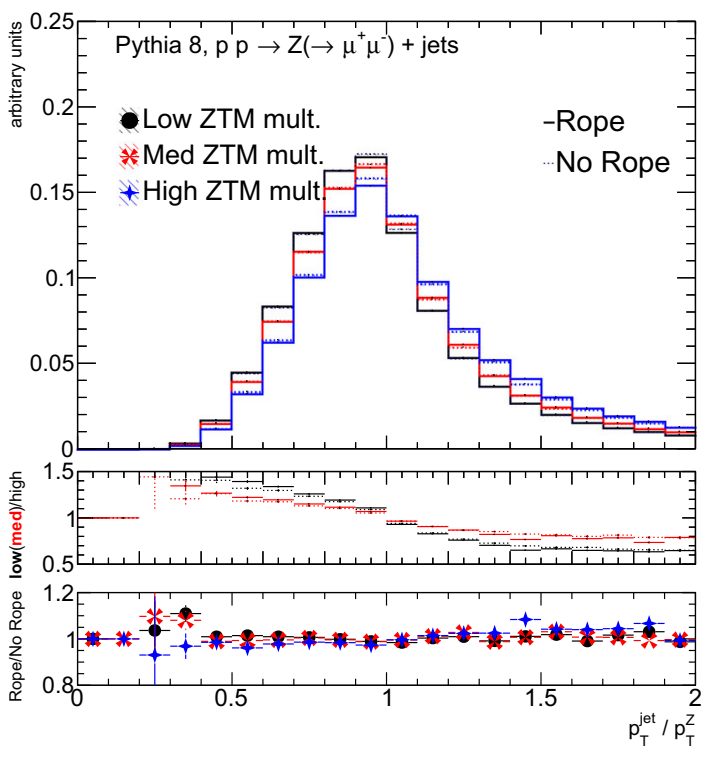

tion with a dotted line). In the lower panel, the ratio between the Rope and standard hadronization models is displayed for all three multiplicity regions 

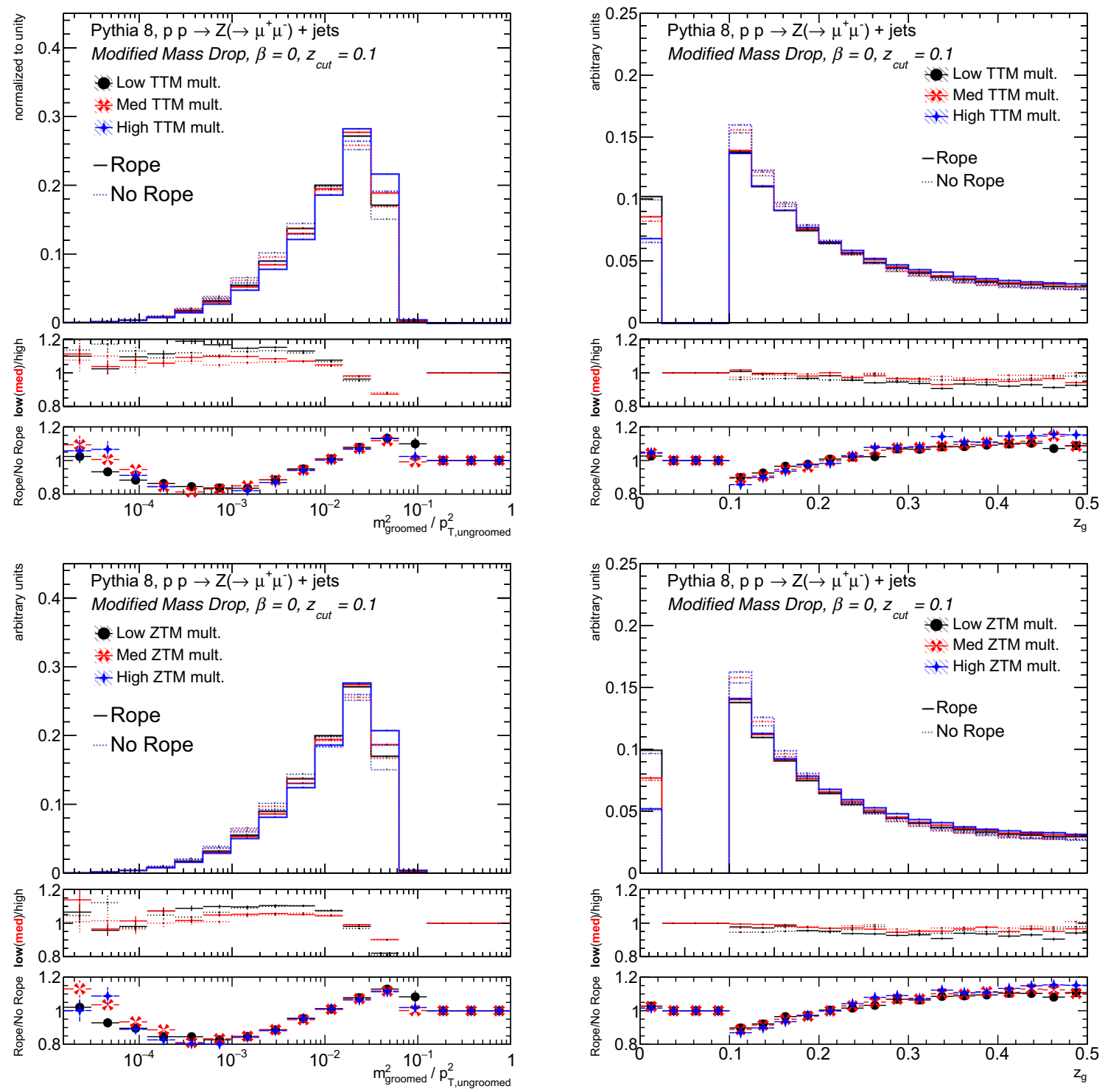

Fig. 20 Modified Mass Drop (also Soft Drop with $\beta=0$ ) jet mass (left) and momentum sharing $z_{g}$ (right) for three bins in FM that correspond to the 50th, 75, and 90th percentiles. The middle panel shows the ratio of the distributions for high to low multiplicity for both hadronization models (standard hadronization with a dotted line). In the lower

panel, the ratio between the Rope and standard hadronization models is displayed for all three multiplicity regions. Due to the algorithm value $z_{\text {cut }}=0.1, z_{g} \geq 0.1$; when the entire jet is groomed away, $z_{g}=0$. The top plots use TTM for the multiplicity while the bottom plots use ZTM 


\section{References}

1. ALICE Collaboration, J. Adam et al., Enhanced production of multi-strange hadrons in high-multiplicity proton-proton collisions. Nat. Phys. 13, 535-539 (2017). arXiv:1606.07424 [nuclex]

2. ATLAS Collaboration, G. Aad et al., Observation of long-range elliptic azimuthal anisotropies in $\sqrt{s}=13$ and $2.76 \mathrm{TeV} p p$ collisions with the ATLAS detector. Phys. Rev. Lett. 116(17), 172301 (2016). arXiv:1509.04776 [hep-ex]

3. CMS Collaboration, Observation of long-range near-side angular correlations in proton-proton collisions at the LHC. JHEP 09, 091 (2010). arXiv:1009.4122 [hep-ex]

4. CMS Collaboration, V. Khachatryan et al., Measurement of longrange near-side two-particle angular correlations in pp collisions at $\sqrt{s}=13 \mathrm{TeV}$. Phys. Rev. Lett. 116(17), 172302 (2016). arXiv: 1510.03068 [nucl-ex]

5. T. Sjöstrand, S. Mrenna, P.Z. Skands, PYTHIA 6.4 physics and manual. JHEP 05, 026 (2006). arXiv:hep-ph/0603175

6. G. Corcella, I.G. Knowles, G. Marchesini, S. Moretti, K. Odagiri, P. Richardson, M.H. Seymour, B.R. Webber, HERWIG 6: an event generator for hadron emission reactions with interfering gluons (including supersymmetric processes). JHEP 01, 010 (2001). arXiv:hep-ph/0011363

7. M. Bahr et al., Herwig++ physics and manual. Eur. Phys. J. C 58, 639-707 (2008). arXiv:0803.0883 [hep-ph]

8. T. Gleisberg, S. Hoeche, F. Krauss, M. Schonherr, S. Schumann, F. Siegert, J. Winter, Event generation with SHERPA 1.1. JHEP 02, 007 (2009). arXiv:0811.4622 [hep-ph]

9. T. Sjöstrand, S. Mrenna, P.Z. Skands, A. Brief, Introduction to PYTHIA 8.1. Comput. Phys. Commun. 178, 852-867 (2008). arXiv:0710.3820 [hep-ph]

10. T. Sjöstrand, S. Ask, J.R. Christiansen, R. Corke, N. Desai, P. Ilten, S. Mrenna, S. Prestel, C.O. Rasmussen, P.Z. Skands, An introduction to PYTHIA 8.2. Comput. Phys. Commun. 191, 159177 (2015). arXiv:1410.3012 [hep-ph]

11. J. Bellm et al., Herwig 7.0/Herwig++ 3.0 release note. Eur. Phys. J. C 76(4), 196 (2016). arXiv:1512.01178 [hep-ph]

12. C. Bierlich, G. Gustafson, L. Lönnblad, A. Tarasov, Effects of overlapping strings in pp collisions. JHEP 03, 148 (2015). arXiv: 1412.6259 [hep-ph]

13. T. Pierog, I. Karpenko, J.M. Katzy, E. Yatsenko, K. Werner, EPOS LHC: test of collective hadronization with data measured at the CERN Large Hadron Collider. Phys. Rev. C 92(3), 034906 (2015). arXiv:1306.0121 [hep-ph]

14. N. Fischer, T. Sjöstrand, Thermodynamical string fragmentation. JHEP 01, 140 (2017). arXiv:1610.09818 [hep-ph]

15. ATLAS Collaboration, G. Aad et al., Observation of associated near-side and away-side long-range correlations in $\sqrt{s_{N N}}=$ 5.02 TeV proton-lead collisions with the ATLAS detector. Phys. Rev. Lett. 110(18), 182302 (2013). arXiv:1212.5198 [hep-ex]

16. ATLAS Collaboration, G. Aad et al., Measurement with the ATLAS detector of multi-particle azimuthal correlations in $\mathrm{p}+\mathrm{Pb}$ collisions at $\sqrt{s_{N N}}=5.02 \mathrm{TeV}$. Phys. Lett. B 725, 60-78 (2013). arXiv:1303.2084 [hep-ex]

17. ATLAS Collaboration, G. Aad et al., Measurement of long-range pseudorapidity correlations and azimuthal harmonics in $\sqrt{s_{N N}}=$ 5.02 TeV proton-lead collisions with the ATLAS detector. Phys. Rev. C 90(4), 044906 (2014). arXiv:1409.1792 [hep-ex]

18. ATLAS Collaboration, G. Aad et al., Centrality and rapidity dependence of inclusive jet production in $\sqrt{s_{\mathrm{NN}}}=5.02 \mathrm{TeV}$ proton-lead collisions with the ATLAS detector. Phys. Lett. B 748, 392-413 (2015). arXiv:1412.4092 [hep-ex]

19. ATLAS Collaboration, G. Aad et al., Measurement of differential $J / \psi$ production cross sections and forward-backward ratios in
$\mathrm{p}+\mathrm{Pb}$ collisions with the ATLAS detector. Phys. Rev. C 92(3), 034904 (2015). arXiv:1505.08141 [hep-ex]

20. ATLAS Collaboration, G. Aad et al., Transverse momentum, rapidity, and centrality dependence of inclusive charged-particle production in $\sqrt{s_{N N}}=5.02 \mathrm{TeV} p+\mathrm{Pb}$ collisions measured by the ATLAS experiment. Phys. Lett. B 763, 313-336 (2016). arXiv:1605.06436 [hep-ex]

21. ATLAS Collaboration, M. Aaboud et al., Measurement of forward-backward multiplicity correlations in lead-lead, protonlead and proton-proton collisions with the ATLAS detector. Phys. Rev. C 95(6), 064914 (2017). arXiv:1606.08170 [hep-ex]

22. ATLAS Collaboration, M. Aaboud et al., Measurements of longrange azimuthal anisotropies and associated Fourier coefficients for $p p$ collisions at $\sqrt{s}=5.02$ and $13 \mathrm{TeV}$ and $p+\mathrm{Pb}$ collisions at $\sqrt{s_{\mathrm{NN}}}=5.02 \mathrm{TeV}$ with the ATLAS detector. arXiv:1609.06213 [nucl-ex]

23. ATLAS Collaboration, M. Aaboud et al., Femtoscopy with identified charged pions in proton-lead collisions at $\sqrt{s_{\mathrm{NN}}}=5.02 \mathrm{TeV}$ with ATLAS (2017). arXiv:1704.01621 [hep-ex]

24. ATLAS Collaboration, M. Aaboud et al., Measurement of multiparticle azimuthal correlations in $p p, p+\mathrm{Pb}$ and low-multiplicity $\mathrm{Pb}+\mathrm{Pb}$ collisions with the ATLAS detector. Eur. Phys. J. C 77(6), 428 (2017). arXiv: 1705.04176 [hep-ex]

25. ATLAS Collaboration, M. Aaboud et al., Measurement of jet fragmentation in $5.02 \mathrm{TeV}$ proton-lead and proton-proton collisions with the ATLAS detector. arXiv:1706.02859 [hep-ex]

26. ATLAS Collaboration, M. Aaboud et al., Measurement of multi-particle azimuthal correlations with the subevent cumulant method in $p p$ and $p+\mathrm{Pb}$ collisions with the ATLAS detector at the LHC. arXiv:1708.03559 [hep-ex]

27. CMS Collaboration, A.M. Sirunyan et al., Measurement of prompt and nonprompt $\mathrm{J} / \psi$ production in $\mathrm{pp}$ and $\mathrm{pPb}$ collisions at $\sqrt{s_{\mathrm{NN}}}=5.02 \mathrm{TeV}$. Eur. Phys. J. C 77(4), 269 (2017). arXiv: 1702.01462 [nucl-ex]

28. C.M.S. Collaboration, A.M. Sirunyan et al., Measurements of the charm jet cross section and nuclear modification factor in $\mathrm{pPb}$ collisions at $\sqrt{s_{N N}}=5.02 \mathrm{TeV}$. Phys. Lett. B 772, 306-329 (2017). arXiv:1612.08972 [nucl-ex]

29. CMS Collaboration, V. Khachatryan et al., Charged-particle nuclear modification factors in $\mathrm{PbPb}$ and $\mathrm{pPb}$ collisions at $\sqrt{s_{\mathrm{N} \mathrm{N}}}=5.02 \mathrm{TeV}$. JHEP 04, 039 (2017). arXiv:1611.01664 [nucl-ex]

30. CMS Collaboration, V. Khachatryan et al., Observation of chargedependent azimuthal correlations in $p$ - $\mathrm{Pb}$ collisions and its implication for the search for the chiral magnetic effect. Phys. Rev. Lett. 118(12), 122301 (2017). arXiv:1610.00263 [nucl-ex]

31. CMS Collaboration, V. Khachatryan et al., Multiplicity and rapidity dependence of strange hadron production in $\mathrm{pp}, \mathrm{pPb}$, and $\mathrm{PbPb}$ collisions at the LHC. Phys. Lett. B 768, 103-129 (2017). arXiv:1605.06699 [nucl-ex]

32. CMS Collaboration, V. Khachatryan et al., Pseudorapidity dependence of long-range two-particle correlations in $p \mathrm{~Pb}$ collisions at $\sqrt{s_{N N}}=5.02 \mathrm{TeV}$. Phys. Rev. C 96(1), 014915 (2017). arXiv: 1604.05347 [nucl-ex]

33. CMS Collaboration, V. Khachatryan et al., Transverse momentum spectra of inclusive $\mathrm{b}$ jets in $\mathrm{pPb}$ collisions at $\sqrt{s_{N N}}=5.02 \mathrm{TeV}$. Phys. Lett. B 754, 59 (2016). arXiv: 1510.03373 [nucl-ex]

34. CMS Collaboration, V. Khachatryan et al., Study of B meson pro-

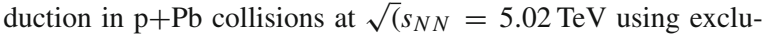
sive hadronic decays. Phys. Rev. Lett. 116(3), 032301 (2016). arXiv:1508.06678 [nucl-ex]

35. CMS Collaboration, V. Khachatryan et al., Evidence for transverse momentum and pseudorapidity dependent event plane fluctuations in $\mathrm{PbPb}$ and $\mathrm{pPb}$ collisions. Phys. Rev. C 92(3), 034911 (2015). arXiv:1503.01692 [nucl-ex] 
36. CMS Collaboration, V. Khachatryan et al., Evidence for collective multiparticle correlations in $\mathrm{p}-\mathrm{Pb}$ collisions. Phys. Rev. Lett. 115(1), 012301 (2015). arXiv:1502.05382 [nucl-ex]

37. CMS Collaboration, S. Chatrchyan et al., Studies of dijet transverse momentum balance and pseudorapidity distributions in $\mathrm{pPb}$ collisions at $\sqrt{s_{\mathrm{NN}}}=5.02 \mathrm{TeV}$. Eur. Phys. J. C 74(7), 2951 (2014). arXiv:1401.4433 [nucl-ex]

38. CMS Collaboration, S. Chatrchyan et al., Event activity dependence of $\mathrm{Y}(\mathrm{nS})$ production in $\sqrt{s_{N N}}=5.02 \mathrm{TeV} \mathrm{pPb}$ and $\sqrt{s}=$ 2.76 TeV pp collisions. JHEP 04, 103 (2014). arXiv:1312.6300 [nucl-ex]

39. CMS Collaboration, S. Chatrchyan et al., Study of the production of charged pions, kaons, and protons in $\mathrm{pPb}$ collisions at $\sqrt{s_{N N}}=$ 5.02 TeV. Eur. Phys. J. C 74(6), 2847 (2014). arXiv:1307.3442 [hep-ex]

40. CMS Collaboration, S. Chatrchyan et al., Multiplicity and transverse momentum dependence of two- and four-particle correlations in $\mathrm{pPb}$ and $\mathrm{PbPb}$ collisions. Phys. Lett. B 724, 213-240 (2013). arXiv:1305.0609 [nucl-ex]

41. CMS Collaboration, S. Chatrchyan et al., Observation of longrange near-side angular correlations in proton-lead collisions at the LHC. Phys. Lett. B 718, 795-814 (2013). arXiv:1210.5482 [nucl-ex]

42. ALICE Collaboration, B. Abelev et al., Pseudorapidity density of charged particles in $p+\mathrm{Pb}$ collisions at $\sqrt{s_{N N}}=5.02 \mathrm{TeV}$. Phys. Rev. Lett. 110(3), 032301 (2013). arXiv:1210.3615 [nucl-ex]

43. ALICE Collaboration, B. Abelev et al., Transverse momentum distribution and nuclear modification factor of charged particles in $p$ - Pb collisions at $\sqrt{s_{N N}}=5.02 \mathrm{TeV}$. Phys. Rev. Lett. 110(8), 082302 (2013). arXiv: 1210.4520 [nucl-ex]

44. ALICE Collaboration, B. Abelev et al., Long-range angular correlations on the near and away side in $p$-Pb collisions at $\sqrt{s_{N N}}=$ 5.02 TeV, Phys. Lett. B 719, 29-41 (2013). arXiv:1212.2001 [nucl-ex]

45. ALICE Collaboration, B.B. Abelev et al., Multiplicity dependence of the average transverse momentum in $\mathrm{pp}, \mathrm{p}-\mathrm{Pb}$, and $\mathrm{Pb}-$ $\mathrm{Pb}$ collisions at the LHC. Phys. Lett. B 727, 371-380 (2013). arXiv:1307.1094 [nucl-ex]

46. ALICE Collaboration, B.B. Abelev et al., Multiplicity dependence of pion, kaon, proton and lambda production in $\mathrm{p}-\mathrm{Pb}$ collisions at $\sqrt{s_{N N}}=5.02 \mathrm{TeV}$. Phys. Lett. B 728, 25-38 (2014). arXiv:1307.6796 [nucl-ex]

47. ALICE Collaboration, B.B. Abelev et al., $J / \psi$ production and nuclear effects in $\mathrm{p}-\mathrm{Pb}$ collisions at $\sqrt{S_{N N}}=5.02 \mathrm{TeV}$. JHEP 02, 073 (2014). arXiv:1308.6726 [nucl-ex]

48. ALICE Collaboration, B.B. Abelev et al., Freeze-out radii extracted from three-pion cumulants in $\mathrm{pp}, \mathrm{pPb}$ and $\mathrm{PbPb}$ collisions at the LHC. Phys. Lett. B 739, 139-151 (2014). arXiv:1404.1194 [nucl-ex]

49. ALICE Collaboration, B.B. Abelev et al., Transverse momentum dependence of inclusive primary charged-particle production in $\mathrm{p}-\mathrm{Pb}$ collisions at $\sqrt{s_{\mathrm{N}}}=5.02 \mathrm{TeV}$. Eur. Phys. J. C 74(9), 3054 (2014). arXiv:1405.2737 [nucl-ex]

50. ALICE Collaboration, B.B. Abelev et al., Measurement of prompt $D$-meson production in $\mathrm{p}-\mathrm{Pb}$ collisions at $\sqrt{s_{N N}}=5.02 \mathrm{TeV}$. Phys. Rev. Lett. 113(23), 232301 (2014). arXiv:1405.3452 [nuclex]

51. ALICE Collaboration, B.B. Abelev et al., Suppression of $\psi(2 \mathrm{~S})$ production in $\mathrm{p}-\mathrm{Pb}$ collisions at $\sqrt{s_{\mathrm{NN}}}=5.02 \mathrm{TeV}$. JHEP 12, 073 (2014). arXiv:1405.3796 [nucl-ex]

52. ALICE Collaboration, B.B. Abelev et al., Multiparticle azimuthal correlations in $\mathrm{p}-\mathrm{Pb}$ and $\mathrm{Pb}-\mathrm{Pb}$ collisions at the CERN Large Hadron Collider. Phys. Rev. C 90(5), 054901 (2014). arXiv:1406.2474 [nucl-ex]

53. ALICE Collaboration, B.B. Abelev et al., Multiplicity dependence of jet-like two-particle correlation structures in $\mathrm{pPb}$ col- lisions at $\sqrt{s_{N N}}=5.02 \mathrm{TeV}$. Phys. Lett. B 741, 38-50 (2015). arXiv: 1406.5463 [nucl-ex]

54. ALICE Collaboration, B.B. Abelev et al., Exclusive $J / \psi$ photoproduction off protons in ultra-peripheral $\mathrm{p}-\mathrm{Pb}$ collisions at $\sqrt{s_{\mathrm{NN}}}=5.02 \mathrm{TeV}$, Phys. Rev. Lett. 113(23), 232504 (2014). arXiv:1406.7819 [nucl-ex]

55. ALICE Collaboration, B.B. Abelev et al., Production of inclusive $\Upsilon(1 \mathrm{~S})$ and $\Upsilon(2 \mathrm{~S})$ in $\mathrm{p}-\mathrm{Pb}$ collisions at $\sqrt{\mathbf{s}_{\mathrm{NN}}}=\mathbf{5 . 0 2} \mathrm{TeV}$. Phys. Lett. B 740, 105-117 (2015). arXiv:1410.2234 [nucl-ex]

56. ALICE Collaboration, J. Adam et al., Centrality dependence of particle production in $\mathrm{p}-\mathrm{Pb}$ collisions at $\sqrt{s_{\mathrm{NN}}}=5.02 \mathrm{TeV}$. Phys. Rev. C 91(6), 064905 (2015). arXiv:1412.6828 [nucl-ex]

57. ALICE Collaboration, J. Adam et al., Two-pion femtoscopy in $\mathrm{p}-\mathrm{Pb}$ collisions at $\sqrt{s_{\mathrm{NN}}}=5.02 \mathrm{TeV}$. Phys. Rev. C 91, 034906 (2015). arXiv:1502.00559 [nucl-ex]

58. ALICE Collaboration, J. Adam et al., Measurement of charged jet production cross sections and nuclear modification in $\mathrm{p}-\mathrm{Pb}$ collisions at $\sqrt{s_{\mathrm{NN}}}=5.02 \mathrm{TeV}$. Phys. Lett. B 749, 68-81 (2015). arXiv: 1503.00681 [nucl-ex]

59. ALICE Collaboration, J. Adam et al., Measurement of dijet $k_{T}$ in pPb collisions at $\sqrt{s_{N N}}=5.02 \mathrm{TeV}$, Phys. Lett. B 746, 385-395 (2015). arXiv:1503.03050 [nucl-ex]

60. ALICE Collaboration, J. Adam et al., Rapidity and transversemomentum dependence of the inclusive $\mathrm{J} / \psi$ nuclear modification factor in p-Pb collisions at $\sqrt{s_{N N}}=5.02 \mathrm{TeV}$. JHEP 06, 055 (2015). arXiv:1503.07179 [nucl-ex]

61. ALICE Collaboration, J. Adam et al., Forward-central twoparticle correlations in $\mathrm{p}-\mathrm{Pb}$ collisions at $\sqrt{s_{\mathrm{NN}}}=5.02 \mathrm{TeV}$. Phys. Lett. B 753, 126-139 (2016). arXiv:1506.08032 [nucl-ex]

62. ALICE Collaboration, J. Adam et al., Centrality dependence of inclusive $\mathrm{J} / \psi$ production in $\mathrm{p}-\mathrm{Pb}$ collisions at $\sqrt{s_{\mathrm{NN}}}=5.02 \mathrm{TeV}$. JHEP 11, 127 (2015). arXiv:1506.08808 [nucl-ex]

63. ALICE Collaboration, J. Adam et al., $\phi$-meson production at forward rapidity in $\mathrm{p}-\mathrm{Pb}$ collisions at $\sqrt{s_{\mathrm{NN}}}=5.02 \mathrm{TeV}$ and in $\mathrm{pp}$ collisions at $\sqrt{s}=2.76 \mathrm{TeV}$. Phys. Lett. B 768, 203-217 (2017). arXiv:1506.09206 [nucl-ex]

64. ALICE Collaboration, J. Adam et al., Multiplicity and transverse momentum evolution of charge-dependent correlations in $\mathrm{pp}, \mathrm{pPb}$, and $\mathrm{PbPb}$ collisions at the LHC. Eur. Phys. J. C 76(2), 86 (2016). arXiv:1509.07255 [nucl-ex]

65. ALICE Collaboration, J. Adam et al., Measurement of electrons from heavy-flavour hadron decays in $\mathrm{p}-\mathrm{Pb}$ collisions at $\sqrt{s_{\mathrm{NN}}}=$ 5.02 TeV. Phys. Lett. B 754, 81-93 (2016). arXiv:1509.07491 [nucl-ex]

66. ALICE Collaboration, J. Adam et al., Multi-strange baryon production in $\mathrm{p}-\mathrm{Pb}$ collisions at $\sqrt{s_{\mathrm{NN}}}=5.02 \mathrm{TeV}$. Phys. Lett. B 758, 389-401 (2016). arXiv:1512.07227 [nucl-ex]

67. ALICE Collaboration, J. Adam et al., Multipion Bose-Einstein correlations in $\mathrm{pp}, \mathrm{p}-\mathrm{Pb}$, and $\mathrm{Pb}-\mathrm{Pb}$ collisions at energies available at the CERN Large Hadron Collider. Phys. Rev. C 93(5), 054908 (2016). arXiv:1512.08902 [nucl-ex]

68. ALICE Collaboration, J. Adam et al., Multiplicity dependence of charged pion, kaon, and (anti)proton production at large transverse momentum in $\mathrm{p}-\mathrm{Pb}$ collisions at $\sqrt{\mathbf{s}_{\mathrm{NN}}}=5.02 \mathrm{TeV}$. Phys. Lett. B 760, 720-735 (2016). arXiv:1601.03658 [nucl-ex]

69. ALICE Collaboration, J. Adam et al., Production of $\mathrm{K}^{*}(892)^{0}$ and $\phi(1020)$ in $\mathrm{pPb}$ collisions at $\sqrt{s_{\mathrm{NN}}}=5.02 \mathrm{TeV}$. Eur. Phys. J. C 76(5), 245 (2016). arXiv:1601.07868 [nucl-ex]

70. ALICE Collaboration, J. Adam et al., Measurement of D-meson production versus multiplicity in $\mathrm{p}-\mathrm{Pb}$ collisions at $\sqrt{\mathrm{s}_{\mathrm{NN}}}=$ 5.02 TeV. JHEP 08, 078 (2016). arXiv:1602.07240 [nucl-ex]

71. ALICE Collaboration, J. Adam et al., Centrality dependence of $\psi(2 \mathrm{~S})$ suppression in $\mathrm{p}-\mathrm{Pb}$ collisions at $\sqrt{\mathbf{s}_{\mathrm{NN}}}=5.02 \mathrm{TeV}$. JHEP 06, 050 (2016). arXiv:1603.02816 [nucl-ex] 
72. ALICE Collaboration, J. Adam et al., Centrality dependence of charged jet production in $\mathrm{pPb}$ collisions at $\sqrt{s_{\mathrm{NN}}}=5.02 \mathrm{TeV}$, Eur. Phys. J. C 76(5), 271 (2016). arXiv:1603.03402 [nucl-ex]

73. ALICE Collaboration, J. Adam et al., Measurement of azimuthal correlations of D mesons and charged particles in pp collisions at $\sqrt{s}=7 \mathrm{TeV}$ and $\mathrm{p}-\mathrm{Pb}$ collisions at $\sqrt{s_{\mathrm{NN}}}=5.02 \mathrm{TeV}$. Eur. Phys. J. C 77(4), 245 (2017). arXiv:1605.06963 [nucl-ex]

74. ALICE Collaboration, J. Adam et al., $D$-meson production in $p$ $\mathrm{Pb}$ collisions at $\sqrt{s_{\mathrm{NN}}}=5.02 \mathrm{TeV}$ and in pp collisions at $\sqrt{s}=$ 7 TeV. Phys. Rev. C 94(5), 054908 (2016). arXiv:1605.07569 [nucl-ex]

75. ALICE Collaboration, J. Adam et al., Measurement of electrons from beauty-hadron decays in $\mathrm{p}-\mathrm{Pb}$ collisions at $\sqrt{s_{\mathrm{NN}}}=$ 5.02 TeV and Pb-Pb collisions at $\sqrt{s_{\mathrm{NN}}}=2.76 \mathrm{TeV}$. JHEP 07, 052 (2017). arXiv:1609.03898 [nucl-ex]

76. ALICE Collaboration, D. Adamova et al., Production of $\Sigma(1385)^{ \pm}$and $\Xi(1530)^{0}$ in $\mathrm{p}-\mathrm{Pb}$ collisions at $\sqrt{s_{\mathrm{NN}}}=5.02 \mathrm{TeV}$. Eur. Phys. J. C 77(6), 389 (2017). arXiv:1701.07797 [nucl-ex]

77. ALICE Collaboration, S. Acharya et al., First measurement of jet mass in $\mathrm{Pb}-\mathrm{Pb}$ and $\mathrm{p}-\mathrm{Pb}$ collisions at the LHC. arXiv: 1702.00804 [nucl-ex]

78. ALICE Collaboration, D. Adamova et al., Azimuthally differential pion femtoscopy in $\mathrm{Pb}-\mathrm{Pb}$ collisions at $\sqrt{s_{\mathrm{NN}}}=2.76 \mathrm{TeV}$. Phys. Rev. Lett. 118(22), 222301 (2017). arXiv:1702.01612 [nuclex]

79. ALICE Collaboration, D. Adamova et al., $\mathrm{J} / \psi$ production as a function of charged-particle pseudorapidity density in $\mathrm{p}-\mathrm{Pb}$ collisions at $\sqrt{s_{\mathrm{NN}}}=5.02 \mathrm{TeV}$. arXiv: 1704.00274 [nucl-ex]

80. ALICE Collaboration, S. Acharya et al., Searches for transverse momentum dependent flow vector fluctuations in $\mathrm{Pb}-\mathrm{Pb}$ and $\mathrm{p}-\mathrm{Pb}$ collisions at the LHC. arXiv:1707.05690 [nucl-ex]

81. LHCb Collaboration, R. Aaij et al., Study of $J / \psi$ production and cold nuclear matter effects in $p P b$ collisions at $\sqrt{s_{N N}}=5 \mathrm{TeV}$. JHEP 02, 072 (2014). arXiv:1308.6729 [nucl-ex]

82. LHCb Collaboration, R. Aaij et al., Study of $\Upsilon$ production and cold nuclear matter effects in $p \mathrm{~Pb}$ collisions at $\sqrt{s_{N N}}=5 \mathrm{TeV}$. JHEP 07, 094 (2014). arXiv:1405.5152 [nucl-ex]

83. LHCb Collaboration, R. Aaij et al., Measurements of long-range near-side angular correlations in $\sqrt{s_{\mathrm{NN}}}=5 \mathrm{TeV}$ proton-lead collisions in the forward region. Phys. Lett. B 762, 473-483 (2016). arXiv:1512.00439 [nucl-ex]

84. LHCb Collaboration, R. Aaij et al., Study of $\psi(2 S)$ production and cold nuclear matter effects in $\mathrm{pPb}$ collisions at $\sqrt{s_{N N}}=5 \mathrm{TeV}$. JHEP 03, 133 (2016). arXiv:1601.07878 [nucl-ex]

85. LHCb Collaboration, R. Aaij et al., Prompt and nonprompt $\mathrm{J} / \psi$ production and nuclear modification in $p \mathrm{~Pb}$ collisions at $\sqrt{s_{\mathrm{NN}}}=$ 8.16 TeV. arXiv:1706.07122 [hep-ex]

86. LHCb Collaboration, R. Aaij et al., Study of prompt $\mathrm{D}^{0}$ meson production in $\mathrm{pPb}$ collisions at $\sqrt{s}=5 \mathrm{TeV}$. arXiv:1707.02750 [hep-ex]

87. B. Andersson, G. Gustafson, G. Ingelman, T. Sjostrand, Parton fragmentation and string dynamics. Phys. Rep. 97, 31-145 (1983)

88. C. Bierlich, Hadronisation models and colour reconnection. PoS DIS 2016, 051 (2016). arXiv: 1606.09456 [hep-ph]

89. C. Bierlich, G. Gustafson, L. Lönnblad, A shoving model for collectivity in hadronic collisions. arXiv:1612.05132 [hep-ph]

90. C. Bierlich, G. Gustafson, L. Lönnblad, Collectivity without plasma in hadronic collisions. arXiv:1710.09725 [hep-ph]

91. A. Ortiz Velasquez, P. Christiansen, E. Cuautle Flores, I. Maldonado Cervantes, G. Paic, Color reconnection and flowlike patterns in $p p$ collisions. Phys. Rev. Lett. 111(4), 042001 (2013). arXiv:1303.6326 [hep-ph]

92. C. Bierlich, J.R. Christiansen, Effects of color reconnection on hadron flavor observables. Phys. Rev. D 92(9), 094010 (2015). arXiv:1507.02091 [hep-ph]
93. J.R. Christiansen, P.Z. Skands, String formation beyond leading colour. JHEP 08, 003 (2015). arXiv:1505.01681 [hep-ph]

94. P. Skands, S. Carrazza, J. Rojo, Tuning PYTHIA 8.1: the Monash 2013 Tune. Eur. Phys. J. C 74(8), 3024 (2014). arXiv:1404.5630 [hep-ph]

95. C. Flensburg, G. Gustafson, L. Lönnblad, Inclusive and exclusive observables from dipoles in high energy collisions. JHEP 08, 103 (2011). arXiv:1103.4321 [hep-ph]

96. M. Cacciari, G.P. Salam, G. Soyez, FastJet User Manual. Eur. Phys. J. C 72, 1896 (2012). arXiv:1111.6097 [hep-ph]

97. M. Cacciari, G.P. Salam, G. Soyez, The anti-k(t) jet clustering algorithm. JHEP 04, 063 (2008). arXiv:0802.1189 [hep-ph]

98. M. Cacciari, G.P. Salam, G. Soyez, The catchment area of jets. JHEP 04, 005 (2008). arXiv:0802.1188 [hep-ph]

99. ALICE Collaboration, B.B. Abelev et al., Performance of the ALICE experiment at the CERN LHC. Int. J. Mod. Phys. A 29, 1430044 (2014). arXiv:1402.4476 [nucl-ex]

100. ATLAS Collaboration, G. Aad et al., The ATLAS experiment at the CERN Large Hadron Collider. JINST 3, S08003 (2008)

101. CMS Collaboration, S. Chatrchyan et al., The CMS Experiment at the CERN LHC. JINST 3, S08004 (2008)

102. ALICE Collaboration, V. Kučera, Production of strange particles in charged jets in $\mathrm{Pb}-\mathrm{Pb}$ and $\mathrm{p}-\mathrm{Pb}$ collisions measured with ALICE. Nucl. Part. Phys. Proc. 276-278, 181-184 (2016). arXiv: 1511.02766 [hep-ex]

103. ATLAS Collaboration, M. Aaboud et al., Jet energy scale measurements and their systematic uncertainties in protonproton collisions at $\sqrt{s}=13 \mathrm{TeV}$ with the ATLAS detector. arXiv: 1703.09665 [hep-ex]

104. CMS Collaboration, Jet energy scale and resolution performances with $13 \mathrm{TeV}$ data, CMS-DP-2016-020 (2016). https://cds.cern.ch/ record $/ 2160347$

105. A.J. Larkoski, S. Marzani, G. Soyez, J. Thaler, Soft Drop. JHEP 05, 146 (2014). arXiv:1402.2657 [hep-ph]

106. M. Dasgupta, A. Fregoso, S. Marzani, G.P. Salam, Towards an understanding of jet substructure. JHEP 09, 029 (2013). arXiv:1307.0007 [hep-ph]

107. A.J. Larkoski, S. Marzani, J. Thaler, Sudakov safety in perturbative QCD. Phys. Rev. D 91(11), 111501 (2015). arXiv:1502.01719 [hep-ph]

108. CMS Collaboration, Splitting function in $\mathrm{pp}$ and $\mathrm{PbPb}$ collisions at $5.02 \mathrm{TeV}$, CMS-PAS-HIN-16-006 (2016). https://cds.cern.ch/ record/2201026

109. Y.-T. Chien, I. Vitev, Probing the hardest branching of jets in heavy ion collisions. arXiv:1608.07283 [hep-ph]

110. Y. Mehtar-Tani, K. Tywoniuk, Groomed jets in heavy-ion collisions: sensitivity to medium-induced bremsstrahlung. JHEP 04, 125 (2017). arXiv:1610.08930 [hep-ph]

111. R. Kunnawalkam Elayavalli, K.C. Zapp, Medium response in JEWEL and its impact on jet shape observables in heavy ion collisions. arXiv:1707.01539 [hep-ph]

112. N.-B. Chang, S. Cao, G.-Y. Qin, Probing medium-induced jet splitting and energy loss in heavy-ion collisions. arXiv: 1707.03767 [hep-ph]

113. J.G. Milhano, U.A. Wiedemann, K.C. Zapp, Sensitivity of jet substructure to jet-induced medium response. arXiv:1707.04142 [hep-ph]

114. STAR Collaboration, K. Kauder, Measurement of the Shared Momentum Fraction $z_{g}$ using Jet Reconstruction in $\mathrm{p}+\mathrm{p}$ and $\mathrm{Au}+\mathrm{Au}$ Collisions with STAR, in 8th International Conference on Hard and Electromagnetic Probes of High-energy Nuclear Collisions: Hard Probes 2016 (HP2016) Wuhan, Hubei, September 23-27, 2016 (2017). arXiv:1703.10933 [nucl-ex]

115. K. Tywoniuk, Is there jet quenching in $\mathrm{pPb}$ ? Nucl. Phys. A 926, 85-91 (2014) 
116. B.G. Zakharov, Parton energy loss in the mini quark-gluon plasma and jet quenching in proton-proton collisions. J. Phys. G41, 075008 (2014). arXiv:1311.1159 [hep-ph]

117. S.-Y. Chen, K.-M. Shen, W. Dai, B.-W. Zhang, H.-Z. Zhang, E.-K. Wang, Centrality dependence of productions for single hadrons and inclusive jets in high-energy $\mathrm{p}+\mathrm{A}$ collisions with NLO QCD. Commun. Theor. Phys. 64(1), 95-102 (2015)
118. Z.-B. Kang, I. Vitev, H. Xing, Effects of cold nuclear matter energy loss on inclusive jet production in $\mathrm{p}+\mathrm{A}$ collisions at energies available at the BNL Relativistic Heavy Ion Collider and the CERN Large Hadron Collider. Phys. Rev. C 92(5), 054911 (2015). arXiv:1507.05987 [hep-ph] 